\title{
Partial Regularity for Harmonic Maps into Spheres at a Singular or Degenerate Free Boundary
}

\author{
Roger Moser $^{1}\left[\right.$ - James Roberts ${ }^{1}$ \\ Received: 15 March 2021 / Accepted: 2 September 2021 / Published online: 5 January 2022 \\ (c) The Author(s) 2021
}

\begin{abstract}
We prove partial regularity of weakly stationary harmonic maps with (partially) free boundary data on manifolds where the domain metric may degenerate or become singular along the free boundary at the rate $d^{\alpha}$ for the distance function $d$ from the boundary.
\end{abstract}

Keywords Harmonic maps · Free boundary · Degenerate elliptic $\cdot$ Partial regularity

Mathematics Subject Classification Primary: 58E20 - 35R35; Secondary: 35B65 . $35 \mathrm{~J} 70$

\section{Introduction}

The regularity of harmonic mappings of Riemannian manifolds with free or constrained boundary data has been increasingly analysed in recent years in view of the connection between these maps and recent definitions of fractional harmonic mappings of Riemannian manifolds, see for example [16,17,19,24]. In order to obtain (partial) regularity for fractional harmonic maps, one is led to the study of free boundary harmonic maps on domains where the Riemannian metric may degenerate or become singular along part of the boundary of the domain, depending on the fractional power in question. The regularity of free boundary harmonic maps, with smooth bounded metrics on the domain, was studied substantially earlier and is of independent interest. For example, free boundary harmonic maps constitute generalisations of minimal surfaces with free boundary. As pointed out in [10], they are also connected with critical points of partially linearised models of elasticity with free boundary conditions and

\footnotetext{
Roger Moser

r.moser@bath.ac.uk

James Roberts

j.e.rob@hotmail.com

1 Department of Mathematical Sciences, University of Bath, Bath BA2 7AY, UK
} 
can also arise in the theory of liquid crystals when modelling surface effects near a solid boundary interface. Motivated by their connection with fractional harmonic maps and the possible other geometric and physical applications, our goal is to establish a partial regularity theory for a class of free boundary harmonic mappings on domains where the metric is conformal to a smooth Riemannian background metric with conformal factor blowing up or vanishing at the boundary. We state some of our results for general target manifolds, but our main theorems concern sphere-valued maps.

Let $\mathcal{M}$ be a smooth Riemannian manifold of dimension $m \geq 3$ with smooth nonempty boundary, equipped with a smooth, bounded Riemannian metric $g$. Suppose $\mathcal{N}$ is a smooth, compact Riemannian manifold which we may assume is isometrically embedded in $\mathbb{R}^{n}$ for some $n \in \mathbb{N}$ due to the theorem of Nash [20]. Let $d:=\operatorname{dist}(\cdot, \partial \mathcal{M})$. We are particularly interested in Riemannian metrics $h$ on $\mathcal{M}$ of the form:

$$
h=d^{\alpha} g
$$

in a neighbourhood of $\partial \mathcal{M}$ for a fixed $\alpha \in\left(-\frac{2}{m-2}, \frac{2}{m-2}\right)$; we will analyse the regularity of a class of critical points $v:(\mathcal{M}, h) \rightarrow \mathbb{R}^{n}$ of the Dirichlet energy on $(\mathcal{M}, h)$ given by

$$
E_{\beta}(v)=\frac{1}{2} \int_{\mathcal{M}}|D v|_{h}^{2} \operatorname{dvol}_{h}=\frac{1}{2} \int_{\mathcal{M}} d^{\beta}|D v|_{g}^{2} \operatorname{dvol}_{g} .
$$

Here, $D$ is the differential of $v, \beta=\frac{\alpha(m-2)}{2} \in(-1,1)$, and $|D v|_{g}^{2}$ is the energy density with respect to $g$. In local coordinates, it is written as $|D v|_{g}^{2}=g^{i j}\left\langle\partial_{i} v, \partial_{j} v\right\rangle$ where $\langle\cdot, \cdot\rangle$ is the Euclidean metric and $\left(g^{i j}\right)=\left(g_{i j}\right)^{-1}$ is the matrix representing $g^{-1}$, and dvol $g$ is the volume form which in local coordinates satisfies $\mathrm{dvol}_{g}=\sqrt{\operatorname{det}(g)} \mathrm{d} x$. Throughout the paper, we assume the convention that repeated indices indicate summation over the appropriate range (unless the index is $m$, which is fixed).

Observe that when $\beta=0$, the energy $E_{0}$ is nothing other than the Dirichlet energy on the manifold $(\mathcal{M}, g)$. In particular, if we were to allow $m=2$ then, regardless of the power $\alpha$, we have $E_{\beta} \equiv E_{0}$; in this case, all the results in this article follow from known results for free boundary harmonic maps, which we will discuss subsequently.

In [24], the second author established partial regularity up to the boundary for minimisers $v: \mathcal{M} \rightarrow \mathcal{N}$ of $E_{\beta}$ with respect to a free boundary condition in the case where $\mathcal{M}$ is a Euclidean half-space. In this article, we will establish partial regularity for maps which are merely critical points of $E_{\beta}$ with respect to both outer and inner variations and still with a free boundary condition. Henceforth, we refer to such maps as weakly stationary harmonic maps with free boundary data.

If we have free boundary data on the whole natural boundary of a manifold, we may find that only constant solutions to the problem exist. As we are studying only the free boundary here, however, we simply exclude any part where the boundary data are not free. Thus, we may imagine that $\mathcal{M}$ is part of a larger manifold $\mathcal{M}^{\prime}$ and $v$ is prescribed on $\mathcal{M}^{\prime} \backslash \mathcal{M}$, but everywhere on $\partial \mathcal{M}$, it is free. As a consequence, we do not assume that $\mathcal{M}$ is complete.

For reasons discussed subsequently, we will also focus on the case where $\mathcal{N}=$ $\mathbb{S}^{n-1} \subset \mathbb{R}^{n}$ is the round unit sphere. An abridged version of our partial regularity theorem states the following. 
Theorem 1.1 Suppose $v: \mathcal{M} \rightarrow \mathbb{S}^{n-1}$ is weakly stationary harmonic with free boundary data. Then there is a closed set $\Sigma \subset \mathcal{M}$ such that $v$ is Hölder continuous in $\mathcal{M} \backslash \Sigma$ and $\mathscr{H}^{m-2}(\Sigma \cap \operatorname{int}(\mathcal{M}))=0$ and $\mathscr{H}^{m+\beta-2}(\Sigma \cap \partial \mathcal{M})=0$.

Here, $\operatorname{int}(\mathcal{M})=\mathcal{M} \backslash \partial \mathcal{M}$ is the interior of $\mathcal{M}$ and $\mathscr{H}^{t}$ is the $t$-dimensional Hausdorff measure. The theorem should properly be formulated for maps in a weighted Sobolev space, with weights behaving like $d^{\beta}$ near the boundary, but we leave the technical details for later.

Away from the boundary $\partial \mathcal{M}$, the regularity stated in the Theorem 1.1 follows from known regularity theory for stationary harmonic maps; these maps are critical points, with respect to both outer and inner variations of the Dirichlet energy for mappings between Riemannian manifolds. Hélein established that weakly harmonic maps on two-dimensional domains are smooth [12]. However, when the dimension of the domain $m$ satisfies $m \geq 3$, harmonic maps are known to have singularities (points of discontinuity) in general and may even be discontinuous on the whole of their domain; Rivière has constructed discontinuous harmonic maps into spheres in [21]. In order to achieve even partial regularity, additional constraints on harmonic maps are required. One way to proceed is to impose geometric constraints, such as the codomain having non-positive sectional curvature. In this case, harmonic maps are also known to be smooth, see [26] for example. In order to deal with general codomain manifolds, however, one must impose a different type of constraint. Typically one considers stationary harmonic maps, or at least maps which satisfy a monotonicity inequality for the re-scaled energy. For such maps, it is possible to estimate the size of the singular set; stationary harmonic maps are smooth in the interior of their domain with the possible exception of a set of vanishing $(m-2)$-dimensional Hausdorff measure. This was first established for sphere-valued maps by Evans [6] and then extended to more general codomain manifolds $\mathcal{N}$ by Bethuel [3].

Near the boundary, when $\beta=0$, the regularity stated in Theorem 1.1 follows from the existing regularity theory for stationary free boundary harmonic maps. As mentioned previously, the regularity theory for such maps was originally investigated with geometric or physical motivations in mind. The regularity of free boundary harmonic maps has been investigated by Baldes [2] and Gulliver and Jost [10] who established full regularity of free boundary harmonic maps under certain geometric constraints, such as the image of the harmonic map lies in a sufficiently small geodesic ball. Scheven later proved partial regularity of free boundary stationary harmonic maps into general target manifolds [25] using a reflection construction in the codomain.

When $\beta \neq 0$, Theorem 1.1 is new. There are related results of the second author [24] (studying minimisers of $E_{\beta}$ ) and of Millot, Pegon and Schikorra [16] who consider a class of stationary or minimising critical points $v: \mathbb{R}^{m-1} \times(0, \infty) \rightarrow \mathbb{R}^{n}$ of $E_{\beta}$, where the domain is the half-space $\mathbb{R}^{m-1} \times(0, \infty)$ and $v$ satisfies the boundary constraint $v(\mathcal{O}) \subset \mathbb{S}^{n-1}$ for some open $\mathcal{O} \subset \partial\left(\mathbb{R}^{m-1} \times(0, \infty)\right)$. Millot, Pegon and Schikorra use the maps they considered as means to establish partial regularity of a type of fractional $s$-harmonic maps into spheres. The maps they consider satisfy $\operatorname{div}\left(x_{m}^{\beta} D v\right)=0$ in a half-space $\mathbb{R}^{m-1} \times(0, \infty)$ together with a non-linear Neumann boundary condition. In contrast we require the maps we consider to satisfy $v(\mathcal{M}) \subset \mathcal{N}$, which makes the condition $v(\mathcal{O}) \subset \mathcal{N}$ redundant. Such maps satisfy a harmonic map equation in the 
interior of the domain and a homogeneous Neumann-type condition on the boundary, see Sect. 3.

We will prove Theorem 1.1 by establishing an $\varepsilon$-regularity result, see Theorem 9.1, and then use a covering argument to conclude the partial regularity stated in Theorem 1.1. The key estimate required to prove Theorem 1.1 is a Caccioppoli-type inequality, see Lemma 8.2. For minimisers, this kind of inequality is established using comparison maps, and we used this approach to prove partial regularity for minimisers of $E_{\beta}$ in [24]. When considering stationary harmonic maps, it is no longer possible to take advantage of the minimising property and a different approach is required; we will instead take advantage of compensated compactness phenomena in the Euler-Lagrange equation.

The underlying observation is that for harmonic maps into a sphere, the expression $|D v|^{2}$ can be written as a product with a 'div-curl' structure. This idea goes back to Hélein, who used it to show regularity of weakly harmonic maps from surfaces into spheres [13]. Evans [6] extended the results of Hélein to higher dimensional domains. The availability of such methods is the reason why we concentrate on spherevalued maps as well. The regularity theory for the usual harmonic maps was further extended to other target manifolds by Bethuel [3], also using concentrated compactness arguments but with different technical details. There is also an alternative approach due to Rivière [22] on two-dimensional domains, which was generalised to higher dimensional domains by Rivière and Struwe [23]. There is no reason a priori why these methods may not be adapted to our setting, too. However, as we wish to avoid the technical difficulties that come with greater generality and concentrate on the new challenges stemming from a Riemannian metric of the form $d^{\alpha} g$, we mostly work with the sphere here.

It has also recently been observed that fractional compensated compactness phenomena appear in the non-local setting for the fractional harmonic maps into spheres considered in [16]. In this paper, as mentioned previously, the maps $v: \mathbb{R}^{m-1} \times$ $(0, \infty) \rightarrow \mathbb{R}^{n}$ satisfy a non-linear Neumann condiditon on the boundary, derived from the boundary constraint $v(\mathcal{O}) \subset \mathcal{N}$ (for $\mathcal{O} \subset \mathbb{R}^{m-1} \times\{0\}$ ). Working directly with the non-local formulation of the boundary condition, Millot, Pegon, and Schikorra showed that this condition may be written as a product with a fractional 'div-curl' structure (plus a controllable error), using fractional notions of divergence and gradient developed by Schikorra and Mazowiecka [15]. Using blow-ups, the authors of [16] also showed that the upper bound for the dimension of the part of the singular set of $v$ contained in $\mathcal{O}$ reduces to the nearest integer when the fractional power in question is non-integer (corresponding to $\beta \neq \frac{1}{2}$ here). In this article, Theorem 1.1 gives a non-integer estimate, namely $m+\beta-2$, on the dimension of part of the singular set of the maps we consider which is contained in the boundary of the domain. This is consistent with the scale invariance of the problem and we expect a similar reduction in dimension for the maps we consider, but for now this is an open question.

One of the cornerstones of the regularity theory for harmonic maps is the duality between BMO spaces and the Hardy spaces $\mathcal{H}^{1}\left(\mathbb{R}^{m}\right)$, combined with estimates for the corresponding norms (see, e.g. the work of Coifman-Lions-Meyer-Semmes [5] or of Chanillo [4], who bypasses this duality but obtains inequalities in the same spirit nevertheless). We will require similar estimates, but adapted to weighted Sobolev spaces, 
which do not appear in the existing literature. The derivation of suitable inequalities will, therefore, occupy a significant portion of this paper.

In the aforementioned regularity theories for harmonic, and fractional harmonic, maps it is known that stationary or minimising maps are smooth away from their singular set. In the context where these regularity theories were developed, higher regularity follows from known estimates for continuous or Hölder continuous solutions to semi-linear elliptic equations, see, respectively, [14] and [27] for example. In [24], the second author studied minimising free boundary harmonic maps in the case where $(\mathcal{M}, g)$ is a half-space with the Euclidean metric. He used related ideas and some additional arguments to show smoothness in directions tangential to free boundary despite the singular factor arising from the factor $d^{\beta}$ in the energy. In this article, the anisotropy of the metric $g$ precludes us from directly applying the theory in [24] unless $g$ is Euclidean (in which case we may draw conclusions on the smoothness of the maps we consider in directions tangential to the boundary as well). Once Hölder continuity is known, we expect minor modifications of the higher regularity results in [24] to yield smoothness for the stationary free boundary harmonic maps we consider here. In particular, we expect all the conclusions of Theorem 4.3 of [24] pertaining to the regularity of the maps considered there, to hold for the maps we consider here (with the exception of the reduced dimension of the singular set in the interior of the domain). However, we will not give the details since we expect all the necessary modifications to be of a purely technical nature. We furthermore observe that there are emerging regularity theories for semi-linear degenerate elliptic equations of the form we consider here, see for example [30]. We note that we could prove a version of Lemma 4.23 in [24] showing Hölder continuity implies Lipschitz continuity and then the results of [30] would apply to give $C^{1, \gamma}$ regularity and possibly even $C^{\infty}$ regularity depending on the precise assumptions required. For the aforementioned reasons, we will not discuss higher regularity any further in this article.

We now outline the organisation of the paper and the strategy for the proof, further details and references may be found at the beginning of each section. In Sect. 2, we introduce local coordinates such that the factor $d^{\beta}$ becomes the weight $x_{m}^{\beta}$ near the boundary; we also introduce the function spaces we require. As discussed previously, the range of specified $\alpha$ implies $\beta \in(-1,1)$. It is then well known that the weight $x_{m}^{\beta}$ then lies in Muckenhoupt class $A_{2}$, see Sect. 7 for the definition, which allows us to apply weighted counterparts to many results, such as the Sobolev-Poincaré inequality, which hold for unweighted Sobolev spaces. In Sect. 3, we give the full definition of stationary harmonic maps with free boundary data and state the corresponding EulerLagrange equations. In Sect. 4, we use the criticality with respect to inner variations to establish energy monotonicity formulas for the re-scaled energy on concentric balls for maps into general target manifolds. We first do this in the coordinates in Sect. 2 and then show that monotonicity inequalities hold independently of the choice of coordinates. We prove control of the $L^{2}$ norm of the average difference between $v$ and its average provided that the re-scaled energy is assumed sufficiently small in Sect. 5, again for general target manifolds. From Sect. 6 onwards, we specialise to the case where the target manifold is a Euclidean sphere. In this case, the Euler-Lagrange equations have a similar structure to the unweighted case, and we show that $d^{\beta}|D v|^{2}$ can be written in 
the form of a weighted 'div-curl' structure. In Sect. 7, we establish weighted versions of the aforementioned compensated compactness results, both globally and locally. We then use the weighted compensated compactness arguments, together with the structure of the Euler-Lagrange equations to establish a Caccioppoli-type inequality for the maps we consider in Sect. 8. With this in hand, it is possible to prove that the re-scaled energy at the boundary decays faster than obtained in the monotonicity formulas described in Sect. 4. We combine this fact with known results in the interior for stationary harmonic maps to obtain $\varepsilon$-regularity and partial regularity in Sect. 9.

Convention We introduce the convention that unless stated otherwise, we write $C$ to denote a constant which depends only on universal factors such as $m, \beta$ or other uniform constants introduced in the next section and we will not always distinguish between different such constants.

\section{Local Coordinates and Function Spaces}

Many of our arguments will make use of local coordinates. As we primarily focus on an analysis of critical points of $E_{\beta}$ near part of the boundary $\partial \mathcal{M}$, it will be convenient to choose local coordinates that map a piece of $\partial \mathcal{M}$ to $\mathbb{R}^{m-1} \times\{0\}$ and at the same time preserve the distance (with respect to $g$ ) to the boundary.

Consider a coordinate chart $x^{\prime}: \mathcal{U}^{\prime} \rightarrow \mathbb{R}^{m-1}$ on $\partial \mathcal{M}$ centred at $x_{0}:=x^{\prime}(p) \in$ $\mathbb{R}^{m-1} \times\{0\}$, where $\mathcal{U}^{\prime} \subset \partial \mathcal{M}$ is a relatively open set. We may choose an open set $\mathcal{U} \subset \mathcal{M}$ such that $\mathcal{U}^{\prime}=\mathcal{U} \cap \partial \mathcal{M}$ and such that the nearest point projection $\pi_{\partial \mathcal{M}}: \mathcal{U} \rightarrow \partial \mathcal{M}$ with respect to $g$ is well defined and smooth in $\mathcal{U}$. Then the distance function $d$ is smooth as well. We define $x: \mathcal{U} \rightarrow \mathbb{R}^{m}$ by

$$
x(q)=\left(x^{\prime}\left(\pi_{\partial M}(q)\right), d(q)\right), \quad q \in \mathcal{U}
$$

i.e. $x=\left(x^{\prime} \circ \pi_{\partial \mathcal{M}}\right) \times d$. This gives rise to local coordinates $x$ on $\mathcal{U}$. If we set $\mathscr{U}=x(\mathcal{U})$, then for $x^{\prime} \in \mathbb{R}^{m-1}$, the lines $\left(\left\{x^{\prime}\right\} \times \mathbb{R}\right) \cap \mathscr{U}$ correspond to geodesics in $\mathcal{U}$ intersecting the boundary perpendicularly, and distances with respect to $g$ are preserved by $x$ along these geodesics. If $\left(g_{i j}\right)$ and $\left(h_{i j}\right)$ denote the components of $g$ and $h$, respectively, in these local coordinates, then

$$
h_{i j}(x)=x_{m}^{\alpha} g_{i j}(x), \quad i, j=1, \ldots, m .
$$

Some of our arguments do not require anything more. However, we will sometimes need to take advantage of some additional properties satisfied by these coordinates. For example, we will prove that a variant of the well-known monotonicity formula for harmonic maps near points of the boundary holds in a uniform way.

Note that the background metric $g$ induces a Riemannian metric $g^{\prime}$ on $\partial \mathcal{M}$ by restriction (even though $h$ does not). Consider a point $p \in \partial \mathcal{M}$ and suppose that $x^{\prime}: \mathcal{U}^{\prime} \rightarrow \mathbb{R}^{m-1}$ describes normal coordinates on $\partial \mathcal{M}$ about $p$ with respect to $g^{\prime}$. If we use the above construction to obtain local coordinates $x: \mathcal{U} \rightarrow \mathbb{R}^{m}$ with this 
choice of $x^{\prime}$, then we automatically find that

$$
g_{i j}\left(x_{0}\right)=\delta_{i j} .
$$

As everything is smooth, it follows that there exist $\rho_{p}>0$ and $c_{p}>0$ such that

$$
\left|g_{i j}(x)-\delta_{i j}\right| \leq c_{p}|x| \text { and }\left|\partial_{k} g_{i j}\right| \leq c_{p} \text { for } i, j, k=1, \ldots, m,
$$

as long as $\left|x-x_{0}\right| \leq \rho_{p}$. Moreover, if $B_{r}^{g}(p)$ denotes the ball of radius $r$ about $p$ with respect to the metric $g$, then for any $r<\rho_{p}$,

$$
B_{r-c_{p} r^{2}}^{g}(p) \subset\left\{q \in \mathcal{M}:\left|x(q)-x_{0}\right|<r\right\} \subset B_{r+c_{p} r^{2}}^{g}(p) .
$$

This gives a useful comparison between balls with respect to $g$ on the one hand and the preimages of Euclidean balls with respect to $x$ on the other hand.

The functions $p \mapsto \rho_{p}$ and $p \mapsto c_{p}$ may be chosen continuous. Hence, for any compact set $\mathcal{K} \subset \mathcal{U}$, there exist $\rho>0$ and $c>0$ such that $\rho_{p} \geq \rho$ and $c_{p} \leq c$ for all $p \in \mathcal{K} \cap \partial \mathcal{M}$. The above inequalities are then uniform in $\mathcal{K}$. Similarly, there exist $C_{0}, \ldots, C_{3}$ such that the inequalities

$$
C_{0}|\xi|^{2} \leq g_{i j} \xi_{i} \xi_{j}, \quad g^{i j} \xi_{i} \xi_{j} \leq C_{1}|\xi|^{2}, \quad\left|g_{i j}\right|,\left|g^{i j}\right| \leq C_{2},
$$

for every $\xi \in \mathbb{R}^{m}$, and

$$
\left|\partial_{k} g_{i j}\right|,\left|\partial_{k} g^{i j}\right| \leq C_{3}
$$

for $k=1, \ldots, m$, hold uniformly in $\mathcal{K}$ even if $x$ is not based on normal coordinates.

We are interested in the regularity of a class of critical points $v: \mathcal{M} \rightarrow \mathcal{N}$ of $E_{\beta}$ such that the energy is, at least locally, finite. We first observe that in the coordinates we have introduced on $\mathscr{U}$ the energy takes the form

$$
\left.E_{\beta}\left|\mathscr{U}(v):=\frac{1}{2} \int_{\mathcal{U}} d^{\beta}\right| D v\right|_{g} ^{2} \operatorname{dvol}_{g}=\frac{1}{2} \int_{\mathscr{U}} x_{m}^{\beta} g^{i j}\left\langle\partial_{i} v, \partial_{j} v\right\rangle \sqrt{\operatorname{det}(g)} \mathrm{d} x .
$$

In order to give meaning to the notion of critical points of $E_{\beta}$ we consider Sobolev spaces on $\mathcal{M}$ such that this expression is, at least locally, finite. We therefore consider function spaces with respect to the weight $x_{m}^{\beta}$ for $\beta \in(-1,1)$. For open $\mathscr{U} \subset \mathbb{R}^{m}$ we denote $L_{\beta}^{p}\left(\mathscr{U} ; \mathbb{R}^{n}\right)$ to be the Banach space of measurable functions $f: \mathscr{U} \rightarrow \mathbb{R}^{n}$ such that the norm $\|f\|_{L_{\beta}^{p}\left(\mathscr{U} ; \mathbb{R}^{n}\right)}:=\left(\int_{\mathscr{U}}\left|x_{m}\right|^{\beta}|f|^{p}\right)^{\frac{1}{p}}$ is finite. Then $W_{\beta}^{1, p}\left(\mathscr{U} ; \mathbb{R}^{n}\right)$ is the normed space of functions $f \in L_{\beta}^{p}\left(\mathscr{U} ; \mathbb{R}^{n}\right)$ with weak derivatives also in $L_{\beta}^{p}\left(\mathscr{U} ; \mathbb{R}^{n}\right)$ and with norm $\|f\|_{W_{\beta}^{1, p}\left(\mathscr{U} ; \mathbb{R}^{n}\right)}:=\left(\int_{\mathscr{U}}\left|x_{m}\right|^{\beta}|f|^{p}+\int_{\mathscr{U}}\left|x_{m}\right|^{\beta}|D f|^{p}\right)^{\frac{1}{p}}$. When $p=2$, the spaces $L_{\beta}^{2}$ and $W_{\beta}^{1,2}$ are Hilbert Spaces with inner products which induce the respective norms. We further define $W_{\beta, 0}^{1, p}\left(\mathscr{U} ; \mathbb{R}^{n}\right)$ as the closure in $W_{\beta}^{1, p}\left(\mathscr{U} ; \mathbb{R}^{n}\right)$ of the space of smooth compactly supported functions $C_{0}^{\infty}\left(\mathscr{U} ; \mathbb{R}^{n}\right)$ on $\mathscr{U}$ with respect to $\|\cdot\|_{W_{\beta}^{1, p}\left(\mathscr{U} ; \mathbb{R}^{n}\right)}$. Since $\left|x_{m}\right|^{\beta}$ is an $A_{2}$ weight, as defined subsequently in (7.1), the 
space of smooth functions in $W_{\beta}^{1, p}\left(\mathscr{U} ; \mathbb{R}^{n}\right)$ is dense in $W_{\beta}^{1, p}\left(\mathscr{U} ; \mathbb{R}^{n}\right)$ for $p \geq 2$ and in this case $W_{\beta}^{1, p}\left(\mathscr{U} ; \mathbb{R}^{n}\right)$ is a Banach space; these properties even hold for $p>q \geq 1$ for some $2>q \geq 1$, but not necessarily all $p \in[1, \infty)$, see [33] Corollary 2.1.6. When $\beta=0$, we omit the subscript in the preceding notation.

Since we are interested in manifold valued maps, we now introduce the function spaces we will use to analyse manifold valued critical points of $E_{\beta}$. We define $W_{\beta}^{1,2}(\mathscr{U} ; \mathcal{N})$ as the collection of maps in $W_{\beta}^{1,2}\left(\mathscr{U} ; \mathbb{R}^{n}\right)$ with values in $\mathcal{N}$ for Lebesgue almost every $x \in \mathscr{U}$. We may then define $W_{\beta, \text { loc }}^{1,2}(\mathcal{M} ; \mathcal{N})$ as maps such that $v \circ x^{-1} \in W_{\beta}^{1,2}(\mathscr{K} ; \mathcal{N})$ for any local coordinates $x: \mathcal{U} \rightarrow \mathscr{U}$ as above and any compact set $\mathscr{K} \subset \mathscr{U}$, as well as $v \in W^{1,2}(\mathcal{K} ; \mathcal{N})$ for every compact set $\mathcal{K} \subset \operatorname{int}(\mathcal{M})$.

We will have occasion to consider a reflection of $v \in W_{\beta}^{1,2}\left(\mathscr{U} ; \mathbb{R}^{n}\right)$ and of the metric $g$ in the hyperplane $\mathbb{R}^{m-1}$. Let $\mathscr{U}_{-}:=\left\{x \in \mathbb{R}^{m}:\left(x^{\prime},-x_{m}\right) \in \mathscr{U}, x_{m}<0\right\}$ and define $\mathscr{V}=\mathscr{U} \cup \mathscr{U}_{-}$. The even reflection of $v$ in the hyperplane $\mathbb{R}^{m-1} \times\{0\}$, which we denote by $\tilde{v}$, satisfies $\tilde{v}(x)=v\left(x^{\prime},\left|x_{m}\right|\right)$, where $x=\left(x^{\prime}, x_{m}\right) \in \mathbb{R}^{m-1} \times \mathbb{R}$ and $\tilde{v} \in W_{\beta}^{1,2}\left(\mathscr{V} ; \mathbb{R}^{n}\right)$. Furthermore, $\partial_{i} \tilde{v}(x)=\partial_{i} v\left(x^{\prime},\left|x_{m}\right|\right)$ for $i \neq m$ and $\partial_{m} \tilde{v}(x)=$ $\operatorname{sgn}\left(x_{m}\right) \partial_{m} v\left(x^{\prime},\left|x_{m}\right|\right)$.

We also extend $g$ to $\mathscr{V}$ by the obvious reflection, giving rise to $\tilde{g}$ on $\mathscr{V}$, and note that $\tilde{g}$ also satisfies (2.4) for the same constants in all of $\mathscr{V}$. It is worthy of note that in the coordinates specified previously in this section, the coefficients $g_{i m}=g_{m i}$ satisfy $g_{i m}\left(x^{\prime}, 0\right)=0$ for $i<m$, and hence, so do the coefficients $g^{i m}\left(x^{\prime}, 0\right)=g^{m i}\left(x^{\prime}, 0\right)$ for $i \neq m$. This means that $\tilde{g}$ and $\tilde{g}^{-1}$ are continuous, and even Lipschitz continuous in compact domains where they are defined. We further note that the eigenvalues of $\tilde{g}^{-1}$ are given by $\lambda_{k}\left(x^{\prime},\left|x_{m}\right|\right)$ where $\lambda_{k}$ are eigenvalues of $g^{-1}$.

\section{The First Variation of $E_{\beta}$}

In this section we specify the class of critical points we consider. We give the definitions in general but only calculate the Euler-Lagrange equations in a chart $\mathscr{U}$ as specified in Sect. 2 since this will suffice for the subsequent analysis.

Let $\pi_{\mathcal{N}}$ denote the nearest point projection on $\mathcal{N}$ and let $v \in W_{\text {loc }}^{1,2}(\mathcal{M} ; \mathcal{N})$. For compact $\mathcal{K} \subset \mathcal{M}$ we define $\left.E_{\beta}\left|\mathcal{K}(v)=\frac{1}{2} \int_{\mathcal{K}} d^{\beta}\right| D v\right|_{g} ^{2} \mathrm{dvol} g$. Observe that $\mathcal{K}$ may intersect the boundary of $\mathcal{M}$. We first consider outer variations of $v$ given by $v_{t}:=$ $\pi_{\mathcal{N}}(v+t \phi) \in W_{\text {loc }}^{1,2}(\mathcal{M} ; \mathcal{N})$ where $\phi \in C_{0}^{\infty}\left(\mathcal{M} ; \mathbb{R}^{n}\right)$. We say that $v \in W_{\text {loc }}^{1,2}(\mathcal{M} ; \mathcal{N})$ is weakly harmonic with respect to free boundary data if for every $\phi \in C_{0}^{\infty}\left(\mathcal{M} ; \mathbb{R}^{n}\right)$ we have $\left.\left.\partial_{t}\right|_{t=0} E_{\beta}\right|_{\operatorname{supp} \phi}\left(v_{t}\right)=0$.

Now let $\mathscr{U}$ be as in Sect. 2 with the given coordinates $x: \mathcal{U} \rightarrow \mathscr{U}$. For $\psi \in$ $C_{0}^{\infty}\left(\mathscr{U} ; \mathbb{R}^{n}\right)$, the function $\phi=\psi \circ x$ gives rise to an admissible outer variation of the form $v_{t}:=\pi_{\mathcal{N}}(v+t \phi)$. Following the computations of e.g [29] Chapter 2, we take the first derivative of $E_{\beta}$ at $t=0$ to see that in the chosen coordinates critical points of $E_{\beta}$ with respect to outer variations satisfy

$$
0=\int_{\mathscr{U}} x_{m}^{\beta} g^{i j}\left(\left\langle\partial_{i} \psi, \partial_{j} v\right\rangle-\left\langle A(v)\left(\partial_{i} v, \partial_{j} v\right), \psi\right\rangle\right) \mathrm{dvol}_{g}
$$


where $A$ is the second fundamental form of $\mathcal{N}$. This is the weak form of the equation

$$
\partial_{i}\left(x_{m}^{\beta} \sqrt{\operatorname{det}(g)} g^{i j} \partial_{j} v\right)+x_{m}^{\beta} \sqrt{\operatorname{det}(g)} g^{i j} A(v)\left(\partial_{i} v, \partial_{j} v\right)=0
$$

in $\mathscr{U}$ with Neumann boundary condition

$$
x_{m}^{\beta} g^{m j} \partial_{j} v=0
$$

on $\mathscr{U} \cap\left(\mathbb{R}^{m-1} \times\{0\}\right)$. More concisely, equation (3.2) can be written in the form

$$
\operatorname{div}_{g}\left(x_{m}^{\beta} \operatorname{grad}_{g} v\right)+x_{m}^{\beta} \operatorname{tr}_{g} A(v)(D v, D v)=0,
$$

where $\operatorname{div}_{g}$, $\operatorname{grad}_{g}$, and $\operatorname{tr}_{g}$ are the divergence, gradient, and trace with respect to $g$, respectively.

The following observation will be useful when we choose certain test functions for (3.2).

Lemma 3.1 If (3.1) is satisfied for all $\psi \in C_{0}^{\infty}\left(\mathscr{U} ; \mathbb{R}^{n}\right)$, then it also holds true for all $\psi \in W_{\beta}^{1,2}\left(\mathscr{U} ; \mathbb{R}^{n}\right) \cap L^{\infty}\left(\mathscr{U} ; \mathbb{R}^{n}\right)$ compactly supported in $\mathscr{U}$.

Proof It is well known that $\left|x_{m}\right|^{\beta}$ is a Muckenhoupt weight of class $A_{2}$, see (7.1) for the definition. By Corollary 2.1.6 in [33] Chapter 2 this implies that $C_{0}^{\infty}\left(\mathscr{U} ; \mathbb{R}^{n}\right)$ is dense in $W_{\beta}^{1,2}\left(\mathscr{U} ; \mathbb{R}^{n}\right)$. Indeed, for $\psi \in W_{\beta}^{1,2}\left(\mathscr{U} ; \mathbb{R}^{n}\right) \cap L^{\infty}\left(\mathscr{U} ; \mathbb{R}^{n}\right)$ with compact support in $\mathscr{U}$, a sequence $\left(\psi_{k}\right)_{k \in \mathbb{N}}$ in $C_{0}^{\infty}\left(\mathscr{U} ; \mathbb{R}^{n}\right)$ converging to $\psi$ can be constructed by convolution with a standard mollifying kernel. Then we also have $\psi_{k} \rightarrow \psi$ pointwise almost everywhere (possibly after passing to a subsequence) and the sequence $\left(\psi_{k}\right)_{k \in \mathbb{N}}$ is bounded in $L^{\infty}\left(\mathscr{U} ; \mathbb{R}^{n}\right)$.

Now it is clear that

$$
\int_{\mathscr{U}} x_{m}^{\beta} g^{i j}\left\langle\partial_{i} \psi, \partial_{j} v\right\rangle \operatorname{dvol}_{g}=\lim _{k \rightarrow \infty} \int_{\mathscr{U}} x_{m}^{\beta} g^{i j}\left\langle\partial_{i} \psi_{k}, \partial_{j} v\right\rangle \mathrm{dvol}_{g} .
$$

Furthermore, the convergence

$$
\int_{\mathscr{U}} x_{m}^{\beta} g^{i j}\left\langle A(v)\left(\partial_{i} v, \partial_{j} v\right), \psi\right\rangle \operatorname{dvol}_{g}=\lim _{k \rightarrow \infty} \int_{\mathscr{U}} x_{m}^{\beta} g^{i j}\left\langle A(v)\left(\partial_{i} v, \partial_{j} v\right), \psi_{k}\right\rangle \mathrm{dvol}_{g}
$$

follows from the Dominated Convergence Theorem. The statement of the lemma now follows immediately.

We now consider inner variations of $E_{\beta}$. Let $\mathcal{K} \subset \mathcal{M}$ be compact and let $\Phi_{t}: \mathcal{M} \rightarrow$ $\mathcal{M}$ constitute a smooth 1-parameter family of diffeomorphisms such that $\Phi_{t}(p)=p$ for all $p \in \mathcal{M} \backslash \mathcal{K}$ and all values of $t$. Then $v_{t}:=v \circ \Phi_{t}$ is an inner variation. Note that $\mathcal{K}$ may include boundary points again. We say that $v$ is weakly stationary harmonic with respect to free boundary data if it is weakly harmonic with respect to free boundary data and $\left.\left.\partial_{t}\right|_{t=0} E_{\beta}\right|_{\mathcal{K}}\left(v_{t}\right)=0$ for all inner variations of the preceding form. 
Consider again local coordinates $x: \mathcal{U} \rightarrow \mathscr{U}$ as in Sect. 2. Let $\Psi_{t}(x)=x+t \psi$ for a $\psi \in C_{0}^{\infty}\left(\mathscr{U} ; \mathbb{R}^{m}\right)$ with $\psi\left(x^{\prime}, 0\right) \subset \mathbb{R}^{m-1} \times\{0\}$ for all $\left(x^{\prime}, 0\right) \in \mathscr{U}$, where $t$ is small enough to make $\Psi_{t}$ a diffeomorphism of $\mathscr{U}$. We apply the above definition to $\Phi_{t}=x^{-1} \circ \Psi_{t} \circ x$. An analysis of $\Psi_{t}$ similar to the standard theory for harmonic maps (see, e.g. [29] again) then shows that a critical point of $E_{\beta}$ with respect to inner variations satisfies

$$
\begin{aligned}
0= & \int_{\mathscr{U}} x_{m}^{\beta} g^{i j}\left(\left\langle\partial_{i} v, \partial_{k} v\right\rangle \partial_{j} \psi_{k}-\frac{1}{2}\left\langle\partial_{i} v, \partial_{j} v\right\rangle \operatorname{div}_{g} \psi\right) \mathrm{dvol}_{g} \\
& -\frac{1}{2} \int_{\mathscr{U}} x_{m}^{\beta} \psi_{k} \partial_{k} g^{i j}\left\langle\partial_{i} v, \partial_{j} v\right\rangle \operatorname{dvol}_{g}-\frac{\beta}{2} \int_{\mathscr{U}} \psi_{m} x_{m}^{\beta-1} g^{i j}\left\langle\partial_{i} v, \partial_{j} v\right\rangle \operatorname{dvol}_{g},
\end{aligned}
$$

where again

$$
\operatorname{div}_{g} \psi=\frac{1}{\sqrt{\operatorname{det}(g)}} \partial_{i}\left(\sqrt{\operatorname{det}(g)} \psi_{i}\right)
$$

is the divergence of $\psi$ with respect to $g$.

This formula is closely related to the observation that the stress-energy tensor of a harmonic map vanishes [1]. (In this case we need to consider the stress-energy tensor with respect to the metric $h$ rather than $g$.) Identity (3.4) gives additional information about the behaviour at the boundary, however.

If any of the criteria defining weakly or stationary harmonic maps with respect to free boundary data are satisfied but with variations only supported away from the boundary, we refer to the maps in question as correspondingly weakly harmonic or weakly stationary harmonic, which is standard terminology.

\section{Monotonicity Formula}

Here we prove a monotonicity formula for half-balls centred on the boundary, similar to that for stationary harmonic maps, using arguments analogous to those of GroßeBrauckmann [9]. Instead of using normal coordinates, we will use those discussed in Sect. 2. The second author proved a version of the following formula in [24], but the present situation is more general in view of the metric $g$. Henceforth, we define $B_{R}^{+}\left(x_{0}\right)=B_{R}\left(x_{0}\right) \cap\left(\mathbb{R}^{m-1} \times(0, \infty)\right)$ where $B_{R}\left(x_{0}\right)=\left\{x \in \mathbb{R}^{m}:\left|x-x_{0}\right|<R\right\}$ is a Euclidean ball. We also write $\partial^{+} \Omega=\partial \Omega \cap\left(\mathbb{R}^{m-1} \times(0, \infty)\right)$ for any $\Omega \subset \mathbb{R}^{m}$.

The following computations take place in local coordinates $x: \mathcal{U} \rightarrow \mathscr{U}$ as discussed in Sect. 2. We now fix these local coordinates and work in $\mathscr{U}$, regarding $g$ as a Riemannian metric on $\mathscr{U}$. We also fix a point $x_{0} \in \mathscr{U} \cap\left(\mathbb{R}^{m-1} \times\{0\}\right)$ and assume that $g_{i j}\left(x_{0}\right)=\delta_{i j}$ and that (2.4) and (2.5) are satisfied.

Lemma 4.1 There exists a constant $C>0$ such that the following holds. Suppose that $v \in W_{\beta}^{1,2}\left(B_{R}^{+}\left(x_{0}\right) ; \mathcal{N}\right)$ satisfies $(3.4)$ for all $\psi \in C_{0}^{\infty}\left(\mathscr{U} ; \mathbb{R}^{m}\right)$ with $\psi\left(x^{\prime}, 0\right) \subset$ $\mathbb{R}^{m-1} \times\{0\}$ for every $\left(x^{\prime}, 0\right) \in \mathscr{U} \cap\left(\mathbb{R}^{m-1} \times\{0\}\right)$. Let $R>0$ such that $B_{2 R}^{+}\left(x_{0}\right) \subset \mathscr{U}$. 
Then for every $0<t<r \leq R$

$$
\begin{aligned}
& 2 \int_{B_{r}^{+}\left(x_{0}\right) \backslash B_{t}^{+}\left(x_{0}\right)} x_{m}^{\beta}\left|x-x_{0}\right|^{2-m-\beta} e^{C\left|x-x_{0}\right|}\left(1+C\left|x-x_{0}\right|\right)^{-1}\left|\frac{\left(x-x_{0}\right)_{i}}{\left|x-x_{0}\right|} \partial_{i} v\right|^{2} \mathrm{dvol}_{g} \\
& \leq r^{2-m-\beta} e^{C r} \int_{B_{r}^{+}\left(x_{0}\right)} x_{m}^{\beta}|D v|_{g}^{2} \mathrm{dvol}_{g}-t^{2-m-\beta} e^{C t} \int_{B_{t}^{+}\left(x_{0}\right)} x_{m}^{\beta}|D v|_{g}^{2} \mathrm{dvol}_{g} .
\end{aligned}
$$

In particular the map $\rho \mapsto \rho^{2-m-\beta} e^{C \rho} \int_{B_{\rho}^{+}\left(x_{0}\right)} x_{m}^{\beta}|D v|_{g}^{2} \mathrm{dvol} g$ is non-decreasing in $\rho$ for $0<\rho<R$.

Proof We may assume without loss of generality that $x_{0}=0$. Let $\eta \in C_{0}^{\infty}\left(B_{R}(0) ; \mathbb{R}\right)$ and define $\psi \in C_{0}^{\infty}\left(B_{R}(0) ; \mathbb{R}^{m}\right)$ by $\psi(x)=x \eta(x)$. Then $\psi_{m}\left(x^{\prime}, 0\right)=0$ so $\psi\left(x^{\prime}, 0\right) \subset \mathbb{R}^{m-1} \times\{0\}$ for every $\left(x^{\prime}, 0\right) \in \mathbb{R}^{m-1} \times\{0\}$. We test (3.4) with $\psi$ and rearrange to see that

$$
\begin{aligned}
(\beta+ & m-2) \int_{B_{R}^{+}(0)} \eta x_{m}^{\beta}|D v|_{g}^{2} \mathrm{dvol}_{g} \\
= & 2 \int_{B_{R}^{+}(0)} x_{m}^{\beta} g^{i j}\left\langle\partial_{i} v, \partial_{k} v\right\rangle x_{k} \partial_{j} \eta \mathrm{dvol}_{g}-\int_{B_{R}^{+}(0)} x_{m}^{\beta}|D v|_{g}^{2} x_{k} \partial_{k} \eta \mathrm{dvol}_{g} \\
& -\int_{B_{R}^{+}(0)} x_{k} \eta x_{m}^{\beta}\left(g^{i j} \frac{\partial_{k} \operatorname{det}(g)}{2 \operatorname{det}(g)}+\partial_{k} g^{i j}\right)\left\langle\partial_{i} v, \partial_{j} v\right\rangle \operatorname{dvol}_{g}
\end{aligned}
$$

Now let $\chi$ be a smooth cutoff function with $\chi \equiv 1$ in $[1, \infty), \chi \equiv 0$ in $(-\infty, 0]$ and such that $0 \leq \chi \leq 1$ and $\left|\chi^{\prime}\right| \leq 3$. Define $\eta_{l}(x)=\chi(l(\rho-|x|))$. Replacing $\eta$ with $\eta_{l}$ in (4.1) and letting $l \rightarrow \infty$ using Lebesgue's Dominated Convergence Theorem and Lebesgue's Differentiation Theorem together we see that

$$
\begin{aligned}
(\beta+ & +2) \int_{B_{\rho}^{+}(0)} x_{m}^{\beta}|D v|_{g}^{2} \mathrm{dvol}_{g} \\
= & -2 \rho^{-1} \int_{\partial^{+} B_{\rho}^{+}(0)} x_{m}^{\beta} g^{i j}\left\langle\partial_{i} v, \partial_{k} v\right\rangle x_{k} x_{j} \mathrm{~d} \mathcal{S}_{g}(\rho)+\rho \int_{\partial^{+} B_{\rho}^{+}(0)} x_{m}^{\beta}|D v|_{g}^{2} \mathrm{~d} \mathcal{S}_{g}(\rho) \\
& -\int_{B_{\rho}^{+}(0)} x_{k} x_{m}^{\beta}\left(g^{i j} \frac{\partial_{k} \operatorname{det}(g)}{2 \operatorname{det}(g)}+\partial_{k} g^{i j}\right)\left\langle\partial_{i} v, \partial_{j} v\right\rangle \operatorname{dvol}_{g}
\end{aligned}
$$

for almost every $\rho<R$, where $\mathrm{d} \mathcal{S}_{g}(\rho)=\sqrt{\operatorname{det}(g)} d \mathcal{H}^{m-1}$ is the surface measure with density $\sqrt{\operatorname{det}(g)}$ relative to the $(m-1)$-dimensional Hausdorff measure.

The function $\rho \mapsto \int_{B_{\rho}^{+}(0)} x_{m}^{\beta}|D v|_{g}^{2} \mathrm{dvol}_{g}$ is differentiable at Lebesgue almost every $\rho \in(0, R)$ with derivative $\int_{\partial^{+} B_{\rho}^{+}(0)} x_{m}^{\beta}|D v|_{g}^{2} \mathrm{~d} \mathcal{S}_{g}(\rho)$. Furthermore, by assumption $g^{i j}(0)=\delta_{i j}$, and thus, we have that $\left|g^{i j}(x) x_{j}-x_{i}\right| \leq C \rho^{2}$. It follows that for almost every $\rho<R$ 


$$
\begin{aligned}
2 \rho & \int_{\partial^{+} B_{\rho}^{+}(0)} x_{m}^{\beta}\left|\frac{x_{i}}{|x|} \partial_{i} v\right|^{2} \mathrm{~d} \mathcal{S}_{g}(\rho) \\
= & (2-m-\beta) \int_{B_{\rho}^{+}(0)} x_{m}^{\beta}|D v|_{g}^{2} \mathrm{dvol}_{g}+\rho \int_{\partial^{+} B_{\rho}^{+}(0)} x_{m}^{\beta}|D v|_{g}^{2} \mathrm{~d} \mathcal{S}_{g}(\rho) \\
& -\int_{B_{\rho}^{+}(0)} x_{k} x_{m}^{\beta}\left(g^{i j} \frac{\partial_{k} \operatorname{det}(g)}{2 \operatorname{det}(g)}+\partial_{k} g^{i j}\right)\left\langle\partial_{i} v, \partial_{j} v\right\rangle \mathrm{dvol}_{g} \\
& -2 \rho^{-1} \int_{\partial^{+} B_{\rho}^{+}(0)} x_{m}^{\beta}\left\langle\partial_{i} v, \partial_{k} v\right\rangle x_{k}\left(g^{i j} x_{j}-x_{i}\right) \mathrm{d} \mathcal{S}_{g}(\rho) \\
\leq & (2-m-\beta+C \rho) \int_{B_{\rho}^{+}(0)} x_{m}^{\beta}|D v|_{g}^{2} \mathrm{dvol} g \\
& +(1+C \rho) \rho \int_{\partial^{+} B_{\rho}^{+}(0)} x_{m}^{\beta}|D v|_{g}^{2} \mathrm{~d} \mathcal{S}_{g}(\rho) \\
\leq & (1+C \rho)\left((2-m-\beta+C \rho) \int_{B_{\rho}^{+}(0)} x_{m}^{\beta}|D v|_{g}^{2} \mathrm{dvol} g\right. \\
& \left.+\rho \int_{\partial^{+} B_{\rho}^{+}(0)} x_{m}^{\beta}|D v|_{g}^{2} \mathrm{~d} \mathcal{S}_{g}(\rho)\right) \\
= & (1+C \rho) e^{-C \rho} \rho_{m+\beta-1} \partial_{\rho}\left(e^{C \rho} \rho^{2-m-\beta} \int_{B_{\rho}^{+}(0)} x_{m}^{\beta}|D v|_{g}^{2} \mathrm{dvol} g\right)
\end{aligned}
$$

for almost every $\rho<R$, where $C$ is a suitable constant. Now we multiply by ( $1+$ $C \rho)^{-1} \rho^{1-m-\beta} e^{C \rho}$ and integrate between $0<t<r<R$ to conclude the proof.

In addition to the monotonicity formula from Lemma 4.1, we can make use of the monotonicity formula derived by Große-Brauckmann [9] for balls in the interior of $\mathcal{M}$. In particular, we use the following consequence of his formula: if $0<t \leq r \leq r_{0}$ and $v$ is a stationary harmonic map with respect to a Riemannian metric $\tilde{g}$ on $B_{r_{0}}^{\tilde{g}}(p)$, where $\tilde{g}$ and its derivatives are uniformly bounded, then $t^{2-m} \int_{B_{t}^{\tilde{g}}(p)}|D v|_{\tilde{g}}^{2} \mathrm{dvol}_{\tilde{g}} \leq$ $C r^{2-m} \int_{B_{r}^{\tilde{g}}(p)}|D v|_{\tilde{g}}^{2} \mathrm{dvol}_{\tilde{g}}$ where $C$ depends only $r_{0}$ and the bounds for $\tilde{g}$ and its first order derivatives. As a consequence, if we have control of the energy in some ball, then we have control of the energy in smaller balls contained therein, too. We now briefly switch our focus to geodesic balls $B_{r}^{g}(p)$ in $\mathcal{M}$ with respect to $g$ in order to emphasise that the corresponding inequalities are independent of the choice of local coordinates. To simplify the notation, we introduce the function

$$
\omega(p, r)=\int_{B_{r}^{g}(p)} d^{\beta} \operatorname{dvol}_{g}, \quad p \in \mathcal{M}, r>0 .
$$

Corollary 4.1 Suppose that $\mathcal{K} \subset \mathcal{M}$ is compact. Then there exist two constants $C, R>$ 0 such that the following holds true. Let $v \in W_{\mathrm{loc}}^{1,2}(\mathcal{M} ; \mathcal{N})$ be a weakly stationary harmonic map with respect to free boundary data. Then for any $p \in \mathcal{K}$ and for $0<t \leq r \leq R$ 


$$
\frac{t^{2}}{\omega(p, t)} \int_{B_{t}^{g}(p)} d^{\beta}|D v|_{g}^{2} \operatorname{dvol}_{g} \leq \frac{C r^{2}}{\omega(p, r)} \int_{B_{r}^{g}(p)} d^{\beta}|D v|_{g}^{2} \operatorname{dvol}_{g} .
$$

Proof We observe that $\omega(p, r)$ is of order $r^{m}$ if $p$ is far away from the boundary and of order $r^{m+\beta}$ for $p \in \partial \mathcal{M}$. Thus, in both cases, the scaling factors in front of the integrals are consistent with Große-Brauckmann's monotonicity formula and Lemma 4.1 , respectively. More generally, we can estimate

$$
\frac{1}{c} \omega(p, r) \leq r^{m}(\max \{r, d(p)\})^{\beta} \leq c \omega(p, r)
$$

throughout $\mathcal{K}$ for some constant $c$.

If $4 t>r$, then the inequality from the lemma is immediate. Therefore, we assume that $4 t \leq r$. Choose $R$ so small that for every $p \in \mathcal{K} \cap \partial \mathcal{M}$, the ball $B_{4 R}(p)$ is contained in a coordinate chart as in Sect. 2.

We first consider $p \in \mathcal{K} \cap \partial \mathcal{M}$ (unless the set is empty). We choose local coordinates $x: \mathcal{U} \rightarrow \mathscr{U}$ with the properties of Sect. 2, including (2.2), and set $x_{0}=x(p) \in \mathscr{U}$. Then Lemma 4.1 applies. Using the notation from the lemma, we conclude that

$$
t^{2-m-\beta} \int_{B_{t}^{+}\left(x_{0}\right)} x_{m}^{\beta}|D v|_{g}^{2} \mathrm{dvol}_{g} \leq C r^{2-m-\beta} \int_{B_{r}^{+}\left(x_{0}\right)} x_{m}^{\beta}|D v|_{g}^{2} \mathrm{dvol}_{g}
$$

for some constant $C$ independent of $p$. Here we work on Euclidean balls depending on the choice of local coordinates. However, according to the discussion in Sect. 2, in particular by (2.3), if we choose $R$ sufficiently small, then

$$
B_{s / 2}^{g}(p) \subset B_{s}^{+}\left(x_{0}\right) \subset B_{2 s}^{g}(p)
$$

for any $s \leq R$. Then

$$
\begin{aligned}
t^{2-m-\beta} \int_{B_{t}^{g}\left(x_{0}\right)} x_{m}^{\beta}|D v|_{g}^{2} \mathrm{dvol}_{g} & \leq t^{2-m-\beta} \int_{B_{2 t}^{+}\left(x_{0}\right)} x_{m}^{\beta}|D v|_{g}^{2} \mathrm{dvol}_{g} \\
& \leq C\left(\frac{r}{2}\right)^{2-m-\beta} \int_{B_{r / 2}^{+}\left(x_{0}\right)} x_{m}^{\beta}|D v|_{g}^{2} \mathrm{dvol}_{g} \\
& \leq C 2^{m+\beta-2} r^{2-m-\beta} \int_{B_{r}^{g}\left(x_{0}\right)} x_{m}^{\beta}|D v|_{g}^{2} \mathrm{dvol}_{g}
\end{aligned}
$$

as required.

If $p \in \mathcal{K}$ satisfies $r / 2 \leq d(p) \leq R$, then we can still work in local coordinates as above, but constructed about a point $p^{\prime} \in \partial \mathcal{M}$. We set $x_{0}=x(p)$ again, which is now in the interior of $\mathscr{U}$. We consider the Riemannian metric $\tilde{h}(x)=r^{-\alpha} h\left(x_{0}+r x\right)$. Then $\tilde{h}$ and all of its derivatives are uniformly bounded in a Euclidean ball $B_{r_{0}}(0)$ for a fixed radius $r_{0}>0$ that is independent of $p$. The map $x \mapsto v\left(x_{0}+r x\right)$ is weakly 
stationary harmonic with respect to the metric $\tilde{h}$, and we can use Große-Brauckmann's monotonicity formula in $B_{r / 4}^{g}(p)$ to derive the estimate

$$
\begin{aligned}
\frac{t^{2}}{\omega(p, t)} \int_{B_{t}^{g}(p)} d^{\beta}|D v|_{g}^{2} \mathrm{dvol}_{g} & \leq \frac{C r^{2}}{\omega(p, r / 4)} \int_{B_{r / 4}^{g}(p)} d^{\beta}|D v|_{g}^{2} \mathrm{dvol}_{g} \\
& \leq \frac{C r^{2}}{\omega(p, r)} \int_{B_{r}^{g}(p)} d^{\beta}|D v|_{g}^{2} \mathrm{dvol}_{g} .
\end{aligned}
$$

For points with $d(p) \geq R$, the behaviour of the metric near the boundary becomes completely irrelevant. In this case, we can use the usual arguments for harmonic maps.

We, hence, consider the case when $d(p)<r / 2$. Here we choose $p^{\prime} \in \partial M$ with $\operatorname{dist}\left(p, p^{\prime}\right)=d(p)$ and we use the inequalities that we already know. Set $s=d(p) / 2$. If $3 t \leq s$, we conclude that

$$
\begin{aligned}
\frac{t^{2}}{\omega(p, t)} \int_{B_{t}^{g}(p)} d^{\beta}|D v|_{g}^{2} \mathrm{dvol}_{g} & \leq \frac{C s^{2}}{\omega(p, s)} \int_{B_{s}^{g}(p)} d^{\beta}|D v|_{g}^{2} \mathrm{dvol}_{g} \\
& \leq \frac{C(3 s)^{2}}{\omega(p, 3 s)} \int_{B_{3 s}^{g}\left(p^{\prime}\right)} d^{\beta}|D v|_{g}^{2} \mathrm{dvol}_{g} \\
& \leq \frac{C(r / 2)^{2}}{\omega(p, r / 2)} \int_{B_{r / 2}^{g}\left(p^{\prime}\right)} d^{\beta}|D v|_{g}^{2} \operatorname{dvol}_{g} \\
& \leq \frac{C r^{2}}{\omega(p, r)} \int_{B_{r}^{g}(p)} d^{\beta}|D v|_{g}^{2} \operatorname{dvol}_{g}
\end{aligned}
$$

If $3 t>s$, then an inequality similar to the first line holds trivially and we can still use the rest of the argument.

When we work with local coordinates, the following version of Corollary 4.1 is convenient. Here we fix the same local coordinates as at the beginning of this section again, and we regard $g$ as a Riemannian metric on the domain $\mathscr{U}$.

Corollary 4.2 There exists a constant $C>0$ with the following property. Let $R>0$ such that $B_{2 R}^{+}\left(x_{0}\right) \subset \mathscr{U}$. Suppose that $v \in W_{\beta}^{1,2}\left(B_{R}^{+}\left(x_{0}\right) ; \mathcal{N}\right)$ is a weakly stationary harmonic map with respect to free boundary data. Let $y \in B_{R / 2}\left(x_{0}\right)$ and $r>0$ such that $B_{r}(y) \subset B_{R}\left(x_{0}\right)$. If $y_{m} \leq r$, then

$$
r^{2-m-\beta} \int_{B_{r}(y) \cap \mathscr{U}} x_{m}^{\beta}|D v|_{g}^{2} \mathrm{dvol}_{g} \leq C R^{2-m-\beta} \int_{B_{R}^{+}\left(x_{0}\right)} x_{m}^{\beta}|D v|_{g}^{2} \mathrm{dvol}{ }_{g} .
$$

If $y_{m} \geq r$, then

$$
r^{2-m} y_{m}^{-\beta} \int_{B_{r}(y) \cap \mathscr{U}} x_{m}^{\beta}|D v|_{g}^{2} \mathrm{dvol}_{g} \leq C R^{2-m-\beta} \int_{B_{R}^{+}\left(x_{0}\right)} x_{m}^{\beta}|D v|_{g}^{2} \mathrm{dvol}_{g} .
$$


Proof For the given local coordinates, Euclidean balls are comparable to geodesic balls with inclusions similar to (2.3). In particular, there exists $c \geq 1$ such that under the above assumptions,

$$
B_{r}(y) \cap \mathscr{U} \subset B_{c r}^{g}(y) \text { and } B_{r}^{g}(y) \subset B_{c r}(y)
$$

Hence,

$$
\int_{B_{r}(y) \cap \mathscr{U}} x_{m}^{\beta}|D v|_{g}^{2} \mathrm{dvol}_{g} \leq C \int_{B_{c r}^{g}(y)} d^{\beta}|D v|_{g}^{2} \mathrm{dvol}_{g}
$$

for a suitable constant $C$. We can then estimate the right-hand side with Corollary 4.1 and, thus, derive the desired inequalities.

Using these monotonicity inequalities we can deduce an estimate in the space of bounded mean oscillation for a stationary weakly harmonic map in terms of the rescaled energy. We recall here that for open $\Omega \subset \mathbb{R}^{m}$, the mean oscillation of a map $v: \Omega \rightarrow \mathbb{R}^{n}$ in $\Omega$ is

$$
[v]_{B M O(\Omega)}=\sup _{B \subset \Omega} \frac{1}{|B|} \int_{B}\left|v-\bar{v}_{B}\right| \mathrm{d} x
$$

where $B \subset \Omega$ is any Euclidean ball, $|B|=\int_{B} \mathrm{~d} x$ and $\bar{v}_{B}=\frac{1}{|B|} \int_{B} v \mathrm{~d} x$. Then $B M O(\Omega):=\left\{v \in L_{l o c}^{1}(\Omega):[v]_{B M O(\Omega)}<\infty\right\}$. We also introduce the notation $|\Omega|_{\beta}=\int_{\Omega}\left|x_{m}\right|^{\beta} \mathrm{d} x$ and $\bar{v}_{\Omega, \beta}=\frac{1}{|\Omega|_{\beta}} \int_{\Omega}\left|x_{m}\right|^{\beta} v \mathrm{~d} x$.

In the following lemma, in addition to the map $v$ on $\mathscr{U}$, we also consider the even reflection $\tilde{v}$ on $\mathscr{V}=\mathscr{U} \cup \mathscr{U}_{-}$as defined in Section 2 .

Lemma 4.2 There exists a constant $C>0$ with the following property. Let $R>0$ such that $B_{2 R}\left(x_{0}\right) \subset \mathscr{V}$. Suppose that $v \in W_{\beta}^{1,2}\left(B_{R}^{+}\left(x_{0}\right) ; \mathcal{N}\right)$ is a weakly stationary harmonic map with respect to free boundary data. Then

$$
[\tilde{v}]_{B M O\left(B_{\frac{R}{2}}\left(x_{0}\right)\right)} \leq C\left(R^{2-m-\beta} \int_{B_{R}^{+}\left(x_{0}\right)} x_{m}^{\beta}|D v|_{g}^{2} \mathrm{dvol}_{g}\right)^{\frac{1}{2}}
$$

Proof We let $B_{r}(y) \subset B_{R / 2}\left(x_{0}\right)$ with centre $y=\left(y^{\prime}, y_{m}\right) \in \mathbb{R}^{m-1} \times \mathbb{R}$. We may assume without loss of generality that $y_{m} \geq 0$. We apply the Poincaré inequality with Hölder's inequality to see that

$$
\begin{aligned}
\frac{1}{\left|B_{r}(y)\right|} \int_{B_{r}(y)}\left|\tilde{v}-\overline{\tilde{v}}_{B_{r}(y)}\right| \mathrm{d} x & \leq C r^{1-m} \int_{B_{r}(y)}|D \tilde{v}| \mathrm{d} x \\
& \leq C r^{1-m}\left(\int_{B_{r}(y)}\left|x_{m}\right|^{-\beta} \mathrm{d} x\right)^{\frac{1}{2}}\left(\int_{B_{r}(y)}\left|x_{m}\right|^{\beta}|D \tilde{v}|^{2} \mathrm{~d} x\right)^{\frac{1}{2}} .
\end{aligned}
$$


Now observe that, similarly to the proof of Corollary 4.1 , for $\beta \in(-1,1)$ we have

$$
\int_{B_{r}(y)}\left|x_{m}\right|^{\beta} \mathrm{d} x \leq C r^{m}\left(\max \left\{r, y_{m}\right\}\right)^{\beta}
$$

for all $\beta \in(-1,1)$, and, hence, in particular for $-\beta$. We apply Corollary 4.2 to complete the proof.

\section{Control of the $L^{2}$-Norm}

Here, and for most of the rest of the paper, we work in local coordinates as in Sect. 2 again. We establish control of the quantity

$$
\int_{B_{r}^{+}\left(x_{0}\right)} x_{m}^{\beta}|v-c|^{2} d x
$$

for certain constants $c$ and suitable radii $r$, for a solution $v$ to the Euler-Lagrange equations for $E_{\beta}$ in terms of the energy in a larger ball, provided the latter energy is assumed small. The control we obtain will allow us to control an $L^{2}$ term which appears in the Caccioppoli-type inequality in Sect. 8 provided the energy is sufficiently small. We establish this control for general target manifolds using a blow-up argument.

First we prove an auxiliary estimate on the energy decay for solutions to the associated linear equations with homogeneous Neumann-type boundary conditions.

Lemma 5.1 Let $\mathscr{U} \subset \mathbb{R}^{m-1} \times[0, \infty)$ be relativelyopen. Let $G=\left(G^{i j}\right): \mathscr{U} \rightarrow \mathbb{R}^{m \times m}$ be a continuous matrix valued function such that $G(x)$ is symmetric and positive definite in $\mathscr{U}$. Suppose further that $G^{i m}\left(x^{\prime}, 0\right)=G^{m i}\left(x^{\prime}, 0\right)=0$ for all $(x, 0) \in$ $\mathscr{U} \cap\left(\mathbb{R}^{m-1} \times\{0\}\right)$ and $i \neq m$. Then for any compact set $\mathscr{K} \subset \mathscr{U}$ there exist constants $C, C^{\prime}, \gamma>0$ with the following properties. Let $y \in \mathbb{R}^{m-1} \times\{0\}$ and $\rho>0$ such that $B_{\rho}^{+}(y) \subset \mathscr{K}$. Suppose $v \in W_{\beta}^{1,2}\left(\mathscr{U} ; \mathbb{R}^{n}\right)$ satisfies

$$
0=\int_{\mathscr{U}} x_{m}^{\beta} G^{i j}\left\langle\partial_{i} v, \partial_{j} \psi\right\rangle \mathrm{d} x
$$

for every $\psi \in C_{0}^{\infty}\left(\mathscr{U} ; \mathbb{R}^{n}\right)$. Then there exists $\lambda \in \mathbb{R}^{n}$ such that

$$
\int_{B_{\rho / 2}^{+}(y)} x_{m}^{\beta}|D v|^{2} \mathrm{~d} x \leq \frac{C}{\rho^{2}} \int_{B_{\rho}^{+}(y)} x_{m}^{\beta}|v-\lambda|^{2} \mathrm{~d} x \leq C^{\prime} \rho^{m+\beta-2+2 \gamma} .
$$

Proof Let $\mathscr{V}=\left\{\left(x^{\prime}, x_{m}\right) \in \mathbb{R}^{m}:\left(x^{\prime},\left|x_{m}\right|\right) \in \mathscr{U}\right\}$. We extend $v$ to $\mathscr{V}$ by even reflection. We also extend $G$ to $\mathscr{V}$, using even reflection of $G^{i j}$ for $1 \leq i, j \leq m-1$ or $i=j=m$ and odd reflection otherwise. Then $v$ is a weak solution of the equation

$$
\partial_{i}\left(\left|x_{m}\right|^{\beta} G^{i j} \partial_{j} v\right)=0 \text { in } \mathscr{V}
$$


Let $\eta \in C_{0}^{\infty}\left(B_{\rho}(y)\right)$ with $\eta \equiv 1$ in $B_{\rho / 2}(y), 0 \leq \eta \leq 1, \eta \equiv 0$ in $\mathbb{R}^{m} \backslash B_{\rho}(y)$ and $|D \eta| \leq \frac{2}{\rho}$. It is well known that $\left|x_{m}\right|^{\beta}$ is a Muckenhoupt weight of class $A_{2}$, see (7.1) for the definition, which yields density of $C^{\infty}\left(B_{\rho}(y) ; \mathbb{R}^{n}\right) \cap W_{\beta}^{1,2}\left(B_{\rho}(y) ; \mathbb{R}^{n}\right)$ in $W_{\beta}^{1,2}\left(B_{\rho}(y) ; \mathbb{R}^{n}\right)$, for instance as a result of Corollary 2.1.6 in [33] Chapter 2. It follows that $\eta^{2}(v-\lambda)$ is an admissible test function for (5.1). Hence, using Young's inequality, we see that

$$
\begin{aligned}
\int_{B_{\rho}^{+}(y)} \eta^{2} x_{m}^{\beta} G^{i j}\left\langle\partial_{i} v, \partial_{j} v\right\rangle \mathrm{d} x= & -2 \int_{B_{\rho}^{+}(y)} x_{m}^{\beta} G^{i j}\left\langle\partial_{i} v, \eta \partial_{j} \eta(v-\lambda)\right\rangle \mathrm{d} x \\
\leq & \frac{1}{2} \int_{B_{\rho}^{+}(y)} \eta^{2} x_{m}^{\beta} G^{i j}\left\langle\partial_{i} v, \partial_{j} v\right\rangle \mathrm{d} x \\
& +C \rho^{-2} \int_{B_{\rho}^{+}(y)} x_{m}^{\beta}|G||v-\lambda|^{2} \mathrm{~d} x
\end{aligned}
$$

In the compact set $\mathscr{K}$ (and in its reflection), we have a uniform upper bound for $G$ and a uniform, positive lower bound for the lowest eigenvalue of $G$. Hence, the first inequality in the statement follows.

For the second inequality, it now suffices to choose $\lambda=v(y)$ and verify that $v$ is Hölder continuous in $\mathscr{K}$. To this end, we apply the regularity theory of [7], in particular Theorem 2.3.12 from [7], to equation (5.3) in $\mathscr{V}$.

Now we prove an estimate for the $L^{2}$-norm of weak solutions of the Euler-Lagrange equations for $E_{\beta}$. Recall the notation

$$
\bar{v}_{\Omega, \beta}=\frac{\int_{\Omega} v\left|x_{m}\right|^{\beta} \mathrm{d} x}{\int_{\Omega}\left|x_{m}\right|^{\beta} \mathrm{d} x}
$$

for $\Omega \subset \mathbb{R}^{m}$. The following result applies to fixed local coordinates as in Sect. 2, as usual, and therefore we assume that $\mathscr{U} \subset \mathbb{R}^{m-1} \times[0, \infty)$ is relatively open and $g$ is a Riemannian metric on $\mathscr{U}$.

Lemma 5.2 Let $\mathscr{K} \subset \mathscr{U}$ be a compact set. For every $\delta, C^{*}>0$ there exist constants $\varepsilon_{0}, \theta_{0} \in\left(0, \frac{1}{8}\right]$ and $R_{0}>0$ such that the following holds. Suppose $v \in W_{\beta}^{1,2}\left(\mathscr{U} ; \mathbb{R}^{n}\right)$ satisfies

$$
\left|\int_{\mathscr{U}} x_{m}^{\beta} g^{i j}\left\langle\partial_{i} \psi, \partial_{j} v\right\rangle \operatorname{dvol}_{g}\right| \leq C^{*} \int_{\mathscr{U}}|\psi| x_{m}^{\beta}|D v|_{g}^{2} \operatorname{dvol}_{g}
$$

for every $\psi \in C_{0}^{\infty}\left(\mathscr{U}, \mathbb{R}^{n}\right)$. Suppose $B_{R}^{+}\left(x_{0}\right) \subset \mathscr{K}$ is a half-ball. If $R \leq R_{0}$ and

$$
R^{2-m-\beta} \int_{B_{R}^{+}\left(x_{0}\right)} x_{m}^{\beta}|D v|_{g}^{2} \mathrm{dvol}_{g} \leq \varepsilon_{0}
$$


then

$$
\left(\theta_{0} R\right)^{-(m+\beta)} \int_{B_{\theta_{0} R}^{+}\left(x_{0}\right)} x_{m}^{\beta}\left|v-\bar{v}_{B_{\theta_{0} R}^{+}\left(x_{0}\right), \beta}\right|^{2} \mathrm{~d} x \leq \delta R^{2-m-\beta} \int_{B_{R}^{+}\left(x_{0}\right)} x_{m}^{\beta}|D v|_{g}^{2} \mathrm{dvol}_{g} .
$$

Proof The proof of the lemma is based on a blow-up procedure, analogous to that of the proof of Lemma 3.5 in [18], for example. Suppose, for a contradiction, that there exist $\delta, C^{*}>0$ such that the claim is false. Then for any $\theta_{0} \in\left(0, \frac{1}{8}\right]$ there is a sequence of maps $\left(v_{k}\right)_{k \in \mathbb{N}}$, with $v_{k} \in W_{\beta}^{1,2}\left(\mathscr{U} ; \mathbb{R}^{n}\right)$ for every $k$, such that

$$
\left|\int_{\mathscr{U}} x_{m}^{\beta} g^{i j}\left\langle\partial_{i} \psi, \partial_{j} v_{k}\right\rangle \mathrm{dvol}_{g}\right| \leq C^{*} \int_{\mathscr{U}}|\psi| x_{m}^{\beta}\left|D v_{k}\right|_{g}^{2} \mathrm{dvol}_{g}
$$

for every $\psi \in C_{0}^{\infty}\left(\mathscr{U} ; \mathbb{R}^{n}\right)$ and a sequence of half-balls $B_{R_{k}}^{+}\left(y_{k}\right) \subset \mathscr{K}$ such that $R_{k} \rightarrow 0^{+}$and

$$
\varepsilon_{k}^{2}:=R_{k}^{2-m-\beta} \int_{B_{R_{k}}^{+}\left(y_{k}\right)} x_{m}^{\beta}\left|D v_{k}\right|_{g}^{2} \mathrm{dvol}_{g} \rightarrow 0 \text { as } k \rightarrow \infty
$$

but

$$
\left(\theta_{0} R_{k}\right)^{-(m+\beta)} \int_{B_{\theta_{0} R_{k}}^{+}\left(y_{k}\right)} x_{m}^{\beta}\left|v_{k}-{\overline{\left(v_{k}\right)}}_{B_{\theta_{0} R_{k}}^{+}\left(y_{k}\right), \beta}\right|^{2} \mathrm{~d} x>\delta \varepsilon_{k}^{2} .
$$

Consider the normalised sequence $\left(w_{k}\right)_{k \in \mathbb{N}}$ with $w_{k} \in W_{\beta}^{1,2}\left(B_{1}^{+}(0) ; \mathbb{R}^{n}\right)$ defined by $w_{k}=\varepsilon_{k}^{-1}\left(v_{k}\left(R_{k} x+y_{k}\right)-{\overline{\left(v_{k}\right)}}_{B_{\theta_{0} R_{k}}^{+}\left(y_{k}\right), \beta}\right)$. Then $\partial_{i} w_{k}(x)=R_{k} \varepsilon_{k}^{-1} \partial_{i} v_{k}\left(R_{k} x+y_{k}\right)$ and, thus,

$$
\int_{B_{1}^{+}(0)} x_{m}^{\beta}\left|D w_{k}\right|^{2} \mathrm{~d} x \leq C \text { and }{\overline{\left(w_{k}\right)}}_{B_{\theta_{0}}^{+}(0), \beta}=0
$$

where $C$ is a constant depending on the Riemannian metric and on $\mathscr{K}$. Furthermore, we deduce from (5.7) that

$$
\theta_{0}^{-(m+\beta)} \int_{B_{\theta_{0}}^{+}(0)} x_{m}^{\beta}\left|w_{k}\right|^{2} \mathrm{~d} x>\delta .
$$

Recall that $\left|x_{m}\right|^{\beta}$ is an $A_{2}$ weight as defined in (7.1). Hence, we may apply (5.8) and the Poincaré inequality for $A_{2}$ weights to deduce that $\left(w_{k}\right)_{k \in \mathbb{N}}$ is bounded in $W_{\beta}^{1,2}\left(B_{1}^{+}(0) ; \mathbb{R}^{n}\right)$. Using Lemma 2.5 of [24], we find a subsequence $\left(w_{k_{l}}\right)_{l \in \mathbb{N}}$ which converges weakly in $W_{\beta}^{1,2}\left(B_{1}^{+}(0) ; \mathbb{R}^{n}\right)$ and strongly in $L_{\beta}^{2}\left(B_{1}^{+}(0) ; \mathbb{R}^{n}\right)$ to a map $w \in$ $W_{\beta}^{1,2}\left(B_{1}^{+}(0) ; \mathbb{R}^{n}\right)$. 
Let $g_{k}=g\left(R_{k} x+y_{k}\right)$. Then in view of (5.6) and (5.8) we calculate

$$
\left|\int_{B_{1}^{+}(0)} x_{m}^{\beta} g_{k}^{i j}\left\langle\partial_{i} w_{k}, \partial_{j} \psi\right\rangle \sqrt{\operatorname{det}\left(g_{k}\right)} \mathrm{d} x\right| \leq C^{*}\|\psi\|_{L^{\infty}\left(B_{1}^{+}(0) ; \mathbb{R}^{n}\right)} \varepsilon_{k}
$$

for every $\psi \in C_{0}^{\infty}\left(B_{1}(0) ; \mathbb{R}^{n}\right)$. Recall that $\mathscr{K}$ is compact. Hence, taking a convergent subsequence of $\left(y_{k_{l}}\right)_{l \in \mathbb{N}}$ and re-indexing, we may assume that $y_{k_{l}} \rightarrow \tilde{y}$, where $\tilde{y}_{m}=0$. Now we observe that the continuity of $g$ yields $g_{k_{l}}(x) \rightarrow F:=g(\tilde{y})$ uniformly.

Using the weak convergence of $\left(w_{k_{l}}\right)_{l \in \mathbb{N}}$ to $w$, it follows that

$$
\begin{aligned}
& \left|\int_{B_{1}^{+}(0)} x_{m}^{\beta} F^{i j}\left\langle\partial_{i} w, \partial_{j} \psi\right\rangle \sqrt{\operatorname{det}(F)} \mathrm{d} x\right| \\
& =\lim _{l \rightarrow \infty}\left|\int_{B_{1}^{+}(0)} x_{m}^{\beta} g_{k_{l}}^{i j}\left\langle\partial_{i} w_{k_{l}}, \partial_{j} \psi\right\rangle \sqrt{\operatorname{det}\left(g_{k_{l}}\right)} \mathrm{d} x\right| \\
& \leq C^{*}\|\psi\|_{L^{\infty}\left(B_{1}^{+}(0) ; \mathbb{R}^{n}\right)} \lim _{l \rightarrow \infty} \varepsilon_{k_{l}}=0
\end{aligned}
$$

for every $\psi \in C_{0}^{\infty}\left(B_{1}(0) ; \mathbb{R}^{n}\right)$. Hence, $w$ satisfies $(5.1)$ from Lemma 5.1 in $B_{1}^{+}(0)$ for the constant function $G=F^{-1} \sqrt{\operatorname{det}(F)}$. We apply the lemma to see that there exist $C>0$ and $\gamma \in(0,1)$ such that

$$
\theta_{0}^{2-m-\beta} \int_{B_{\theta_{0}}^{+}(0)} x_{m}^{\beta}|D w|^{2} \mathrm{~d} x \leq C \theta_{0}^{2 \gamma}
$$

We also conclude, using strong convergence of $\left(w_{k_{l}}\right)_{l \in \mathbb{N}}$ to $w$ in $L_{\beta}^{2}\left(B_{1}^{+}(0) ; \mathbb{R}^{n}\right)$ to take limits in (5.9), that

$$
\theta_{0}^{-(m+\beta)} \int_{B_{\theta_{0}}^{+}(0)} x_{m}^{\beta}|w|^{2} \mathrm{~d} x \geq \delta
$$

Now, since $\bar{w}_{B_{\theta_{0}}^{+}(0), \beta}=0$, the Poincaré inequality for $A_{2}$ weights yields

$$
\theta_{0}^{-(m+\beta)} \int_{B_{\theta_{0}}^{+}(0)} x_{m}^{\beta}|w|^{2} \mathrm{~d} x \leq C \theta_{0}^{2-m-\beta} \int_{B_{\theta_{0}}^{+}(0)} x_{m}^{\beta}|D w|^{2} \mathrm{~d} x .
$$

Inequalities (5.10), (5.11), and (5.12) finally imply that $\delta \leq C \theta_{0}^{2 \gamma}$. If $\theta_{0}$ is chosen sufficiently small, then this is a contradiction.

\section{Maps into Spheres}

All of our results in previous sections apply to (free boundary) harmonic maps into a general smooth compact manifold $\mathcal{N}$. In this section and henceforth, we specialise 
to the case where $\mathcal{N}=\mathbb{S}^{n-1}=\left\{y \in \mathbb{R}^{n}:|y|=1\right\}$ is the round $n$-sphere embedded isometrically in $\mathbb{R}^{n}$. In this case a weakly harmonic map $v \in W_{\beta}^{1,2}\left(\mathscr{U} ; \mathbb{S}^{n-1}\right)$ with respect to free boundary data satisfies

$$
0=\int_{\mathscr{U}} x_{m}^{\beta}\left(g^{i j}\left\langle\partial_{i} \psi, \partial_{j} v\right\rangle-|D v|_{g}^{2}\langle v, \psi\rangle\right) \mathrm{dvol}_{g}
$$

for every $\psi \in C_{0}^{\infty}\left(\mathscr{U} ; \mathbb{R}^{n}\right)$.

In order to establish partial regularity, as in the case for stationary harmonic maps into spheres, we take advantage of the fact that the Euler-Lagrange equations can be rewritten in a form reminiscent of a conservation law. We write (6.1) essentially in 'div-curl' form but we also have boundary conditions coming from the free boundary.

Lemma 6.1 A map $v \in W_{\beta}^{1,2}\left(\mathscr{U} ; \mathbb{S}^{n-1}\right)$ satisfies $(6.1)$ for every $\psi \in C_{0}^{\infty}\left(\mathscr{U} ; \mathbb{R}^{n}\right)$ if and only if the vector fields $X_{a b} \in L_{-\beta}^{2}\left(\mathscr{U} ; \mathbb{R}^{m}\right)$ defined in components by

$$
X_{a b}^{i}=x_{m}^{\beta} \sqrt{\operatorname{det}(g)} g^{i j}\left(v^{a} \partial_{j} v^{b}-v^{b} \partial_{j} v^{a}\right)
$$

satisfy

$$
\int_{\mathscr{U}} \partial_{i} \psi X_{a b}^{i} \mathrm{~d} x=0
$$

for $a, b=1, \ldots, n$ and every $\psi \in C_{0}^{\infty}(\mathscr{U} ; \mathbb{R})$.

Remark Lemma 6.1 asserts that $v \in W_{\beta}^{1,2}\left(\mathscr{U} ; \mathbb{S}^{n-1}\right)$ is weakly harmonic with respect to free boundary data if and only if the vector fields $X_{a b} \in L_{-\beta}^{2}\left(\mathscr{U} ; \mathbb{R}^{n}\right)$ satisfy the equation $\operatorname{div}\left(X_{a b}\right)=0$ in $\operatorname{int}(\mathscr{U})$ with boundary condition $X_{a b}=0$ on $\mathscr{U} \cap\left(\mathbb{R}^{m-1} \times\right.$ $\{0\})$ weakly.

Proof We follow the proof of Lemma 3.5 in [18]. Suppose $v \in W_{\beta}^{1,2}\left(\mathscr{U} ; \mathbb{S}^{n-1}\right)$ satisfies (6.1) for every $\psi \in C_{0}^{\infty}\left(\mathscr{U} ; \mathbb{R}^{n}\right)$. Then, by Lemma 3.1, $v$ satisfies (6.1) for every $\psi \in W_{\beta}^{1,2}\left(\mathscr{U} ; \mathbb{R}^{n}\right) \cap L^{\infty}\left(\mathscr{U} ; \mathbb{R}^{n}\right)$ with support compactly contained in $\mathscr{U}$. Then, for $\psi \in C_{0}^{\infty}(\mathscr{U} ; \mathbb{R})$, we conclude that the map $\psi v^{a} \in W_{\beta}^{1,2}(\mathscr{U} ; \mathbb{R}) \cap L^{\infty}(\mathscr{U} ; \mathbb{R})$ is an admissible test function and it follows that

$$
\begin{aligned}
\int_{\mathscr{U}} \partial_{i} \psi X_{a b}^{i} \mathrm{~d} x & =\int_{\mathscr{U}} x_{m}^{\beta} g^{i j}\left(\partial_{i}\left(\psi v^{a}\right) \partial_{j} v^{b}-\partial_{i}\left(\psi v^{b}\right) \partial_{j} v^{a}\right) \mathrm{dvol}_{g} \\
& =\int_{\mathscr{U}} x_{m}^{\beta}|D v|_{g}^{2} \psi\left(v^{a} v^{b}-v^{a} v^{b}\right) \mathrm{dvol}_{g}=0 .
\end{aligned}
$$

Conversely, suppose that (6.3) is satisfied for $a, b=1, \ldots, n$ and every $\psi \in$ $C_{0}^{\infty}(\mathscr{U} ; \mathbb{R})$. Now let $\psi \in C_{0}^{\infty}\left(\mathscr{U} ; \mathbb{R}^{n}\right)$ with components $\psi^{b} \in C_{0}^{\infty}(\mathscr{U} ; \mathbb{R})$. Recall that for each $i=1, \ldots, m$ we have $v^{a} \partial_{i} v^{a}=0$ almost everywhere since $|v|^{2}=1$ almost everywhere. Furthermore, observe that by approximation $v^{a} \psi^{b}$ is an admissible test function for (6.3) for every $a, b=1, \ldots, n$. Hence, summing over $a, b=1, \ldots, n$, we find 


$$
\begin{aligned}
0 & =\int_{\mathscr{U}} \partial_{i}\left(v^{a} \psi^{b}\right)\left(x_{m}^{\beta} \sqrt{\operatorname{det}(g)} g^{i j}\left(v^{a} \partial_{j} v^{b}-v^{b} \partial_{j} v^{a}\right)\right) \mathrm{d} x \\
& =\int_{\mathscr{U}} x_{m}^{\beta} \sqrt{\operatorname{det}(g)} g^{i j}\left(\psi^{b} \partial_{i} v^{a}\left(v^{a} \partial_{j} v^{b}-v^{b} \partial_{j} v^{a}\right)+v^{a} \partial_{i} \psi^{b}\left(v^{a} \partial_{j} v^{b}-v^{b} \partial_{j} v^{a}\right)\right) \mathrm{d} x \\
& =\int_{\mathscr{U}} x_{m}^{\beta} g^{i j}\left(\left\langle\partial_{i} \psi, \partial_{j} v\right\rangle-\left\langle\partial_{i} v, \partial_{j} v\right\rangle\langle v, \psi\rangle\right) \mathrm{dvol}_{g}
\end{aligned}
$$

as required.

\section{Hardy Space Estimate with Weights}

In order to establish partial regularity for sphere-valued stationary harmonic maps we follow the approach in [18] Section 3 (based on ideas by Hélein [13] and Evans [6]). In particular, we combine the estimate given by Lemma 5.2 for the mean squared oscillation with a Caccioppoli-Type inequality to show that if the re-scaled energy is sufficiently small, then it decays faster than implied by the monotonicity formula in Lemma 4.1. In order to derive this inequality, we will take advantage of the equation derived in Lemma 6.1 satisfied by $v$. It implies, roughly speaking, that the product $D v \cdot X_{a b}$ lies, after extending by a reflection with respect to $\mathbb{R}^{m-1} \times\{0\}$, in a Hardy space.

We recall the definition of the Hardy space $\mathcal{H}^{1}\left(\mathbb{R}^{m}\right)$. For more details, see [31], for example. Let $\mathcal{F}$ be the collection of all $\psi \in C_{0}^{\infty}\left(B_{1}(0) ; \mathbb{R}\right)$ with $\sup _{B_{1}(0)}|D \psi| \leq$ 1. Define $\psi_{s}(x)=s^{-m} \psi\left(s^{-1} x\right)$ for $\psi \in \mathcal{F}$ and $s>0$. For a locally integrable distribution $v$ on $\mathbb{R}^{m}$ let $\psi_{s} * v=\int_{\mathbb{R}^{m}} \psi_{s}(x-y) v(y) \mathrm{d} y$ and define the maximal function

$$
M v(x)=\sup _{\psi \in \mathcal{F}} \sup _{s>0}\left|\left(\psi_{s} * v\right)(x)\right|
$$

for $x \in \mathbb{R}^{m}$. The Hardy Space $\mathcal{H}^{1}\left(\mathbb{R}^{m}\right)$ then consists of locally integrable distributions $v$ with norm $\|v\|_{\mathcal{H}^{1}\left(\mathbb{R}^{m}\right)}=\|M v\|_{L^{1}\left(\mathbb{R}^{m}\right)}<\infty$.

In order to prove our Hardy space estimate, we will need to utilise the theory of $A_{p}$ weights, which we define and discuss presently. Suppose $\mu$ and $v$ are measures and let $\mathrm{d} \nu=w \mathrm{~d} \mu$ for a non-negative locally integrable function $w$. Then $w$ is in $A_{p}(\mathrm{~d} \mu)$ if there exists a constant $c>0$ such that

$$
\frac{1}{\mu(B)} \int_{B} w \mathrm{~d} \mu\left(\frac{1}{\mu(B)} \int_{B} w^{-\frac{1}{p-1}} \mathrm{~d} \mu\right)^{p-1} \leq c
$$

for every ball $B \subset \mathbb{R}^{m}$, see [32] Chapter I.

For the $A_{p}$ class of weights, many counterparts to estimates in unweighted Sobolev-Spaces remain true. We recall the estimates we need here. Let $\mathrm{d} x$ denote the $m$-dimensional Lebesgue measure. We use the notation $W^{1, p}(\mathscr{U} ; w \mathrm{~d} x)$ to denote weighted Sobolev spaces comprised of classes of $\mathrm{d} x$ measurable functions with weak first order derivatives such that the function and all its first order derivatives are $p$ integrable with respect to the measure $w \mathrm{~d} x$. Recall that smooth functions contained 
in $W^{1, p}(\mathscr{U} ; w \mathrm{~d} x)$ are dense provided $w$ is an $A_{p}$ weight, see Corollary 2.1.6 of [33]. We further have the following Sobolev-Poincaré inequality.

Lemma 7.1 ([7] Theorem 1.5) Let $w \in A_{p}(\mathrm{~d} x)$ where $1<p<\infty$. Then there exist $C, \delta>0$ such that for $1 \leq \kappa \leq \frac{m}{m-1}+\delta$ and any $v$ which is Lipschitz continuous on the closure of $B_{r}\left(x_{0}\right) \subset \mathbb{R}^{m}$ we have

$$
\left(\frac{1}{\int_{B_{r}\left(x_{0}\right)} w \mathrm{~d} x} \int_{B_{r}\left(x_{0}\right)}|v-\bar{v}|^{\kappa p} w \mathrm{~d} x\right)^{\frac{1}{\kappa p}} \leq C r\left(\frac{1}{\int_{B_{r}\left(x_{0}\right)} w \mathrm{~d} x} \int_{B_{r}\left(x_{0}\right)}|D v|^{p} w \mathrm{~d} x\right)^{\frac{1}{p}}
$$

where we may choose either $\bar{v}=\frac{1}{\int_{B_{r}\left(x_{0}\right)} w \mathrm{~d} x} \int_{B_{r}\left(x_{0}\right)} v w \mathrm{~d} x$ or $\bar{v}=\frac{1}{\int_{B_{r}\left(x_{0}\right)} \mathrm{d} x} \int_{B_{r}\left(x_{0}\right)} v \mathrm{~d} x$. Furthermore, (7.2) extends by density to hold for $v \in W^{1, p}\left(B_{r}\left(x_{0}\right) ; w \mathrm{~d} x\right)$ by Corollary 2.1.6 of [33].

We observe that the bound $\frac{m}{m-1}+\delta$ may not allow the unweighted Sobolev exponent $\kappa=\frac{m}{m-p}$; however, the bound given in the Lemma is sufficient for our purpose. A more precise bound is given in Chapter 15 of [11].

We define the Hardy-Littlewood maximal operator corresponding to a measure $\mathrm{d} \mu$ by $M_{\mu}(f)(x)=\sup _{B \ni x} \frac{1}{\mu(B)} \int_{B}|f| \mathrm{d} \mu$. For $A_{p}$ weights, the following version of the Hardy-Littlewood Maximal Theorem holds.

Lemma 7.2 ([32] Theorem 9) Let $p>1$ and suppose $\mathrm{d} v=w \mathrm{~d} \mu$ where $w \in A_{p}(\mathrm{~d} \mu)$. Let $M_{\mu}$ denote the Hardy Maximal function associated to $\mu$. Then $\left\|M_{\mu}(f)\right\|_{L^{p}(\mathrm{~d} v)} \leq$ $C_{p}\|f\|_{L^{p}(\mathrm{~d} v)}$ for every $f \in L^{p}(\mathrm{~d} v)$.

Now we are in a position to prove our weighted Hardy estimates. We first establish an estimate for the product of a gradient and a quantity which is divergence free in $\mathbb{R}^{m}$. We use $\cdot$ to denote the inner product in $\mathbb{R}^{m}$ for brevity.

Lemma 7.3 Let $\beta \in(-1,1)$ and $m \geq 3$. Then there exists $C>0$ such that if $v \in W_{\beta}^{1,2}\left(\mathbb{R}^{m}\right)$ and $X \in L_{-\beta}^{2}\left(\mathbb{R}^{m} ; \mathbb{R}^{m}\right)$ satisfies $\operatorname{div}(X)=0$ weakly in $\mathbb{R}^{m}$ then $D v \cdot X \in \mathcal{H}^{1}\left(\mathbb{R}^{m}\right)$ and

$$
\|D v \cdot X\|_{\mathcal{H}^{1}\left(\mathbb{R}^{m}\right)} \leq C\|D v\|_{L_{\beta}^{2}\left(\mathbb{R}^{m}\right)}\|X\|_{L_{-\beta}^{2}\left(\mathbb{R}^{m}\right)}
$$

Remark We will require certain weight functions to satisfy corresponding $A_{p}$ conditions for the proof of Lemma 7.3 but postpone the proof of these conditions to a subsequent Lemma, see Lemma 7.5.

Proof of Lemma 7.3 The following arguments are inspired by the work of CoifmanLions-Meyer-Semmes [5]. 
Let $\psi_{s}(x)=s^{-m} \psi\left(\frac{x}{s}\right)$ for $s>0$ and where $\psi \in C_{0}^{\infty}\left(B_{1}(0) ; \mathbb{R}\right)$ with $|D \psi| \leq 1$. Then, for any $\bar{v} \in \mathbb{R}^{n}$, we have

$$
\begin{aligned}
\psi_{s} *(D v \cdot X) & =s^{-m} \int_{B_{s}(x)} \psi\left(\frac{x-y}{s}\right) D v \cdot X \mathrm{~d} y \\
& =-s^{-m-1} \int_{B_{S}(x)} D \psi\left(\frac{x-y}{s}\right)(v-\bar{v}) \cdot X \mathrm{~d} y .
\end{aligned}
$$

Now fix $q \in\left(2, \frac{2 m}{m-1}\right)$. Then $2>\frac{q}{q-1}>1$ and $2>q \frac{m-1}{m}>1$. Furthermore, we check that

$$
(\beta+1) \frac{(m-1)}{2 m}-\frac{1}{q}<\min \left\{\frac{1-\frac{2}{q}+\beta}{2}, 1-\frac{m+q}{m q}\right\} .
$$

Hence, we may choose $\gamma \neq 0$ such that

$$
(\beta+1) \frac{(m-1)}{2 m}-\frac{1}{q}<\gamma<\min \left\{\frac{1-\frac{2}{q}+\beta}{2}, 1-\frac{m+q}{m q}\right\} .
$$

Let $\bar{v}$ be the average of $v$ on $B_{S}(x)$ with respect to $\left|y_{m}\right|^{\gamma q} \mathrm{~d} y$. Furthermore, we write

$$
|\Omega|_{\sigma}=\int_{\Omega}\left|y_{m}\right|^{\sigma} d y
$$

for measurable sets $\Omega \subset \mathbb{R}^{m}$ and we omit the subscript when $\sigma=0$. We have

$$
-s^{-m-1} \int_{B_{s}(x)} D \psi\left(\frac{x-y}{s}\right)(v-\bar{v}) \cdot X \mathrm{~d} y \leq \frac{C}{s\left|B_{s}(x)\right|} \int_{B_{s}(x)}|v-\bar{v}||X| \mathrm{d} y .
$$

Then, using Hölder's inequality for the Lebesgue measure we see that,

$$
\begin{aligned}
& \frac{C}{s\left|B_{S}(x)\right|} \int_{B_{S}(x)}|v-\bar{v}||X| \mathrm{d} y \\
& =\frac{C}{s\left|B_{S}(x)\right|} \int_{B_{s}(x)}\left|y_{m}\right|^{\gamma}\left|y_{m}\right|^{-\gamma}|v-\bar{v}||X| \mathrm{d} y \\
& \leq \frac{C}{s\left|B_{S}(x)\right|}\left(\int_{B_{S}(x)}\left|y_{m}\right|^{q \gamma}|v-\bar{v}|^{q} \mathrm{~d} y\right)^{\frac{1}{q}}\left(\int_{B_{S}(x)}\left|y_{m}\right|^{-\gamma \frac{q}{q-1}}|X|^{\frac{q}{q-1}} \mathrm{~d} y\right)^{\frac{q-1}{q}} \\
& =\frac{C\left|B_{S}(x)\right|_{\gamma q}^{\frac{1}{q}}\left|B_{S}(x)\right|_{-\gamma \frac{q}{q-1}}^{\frac{q-1}{q}}}{s\left|B_{S}(x)\right|}\left(\frac{1}{\left|B_{S}(x)\right|_{\gamma q}} \int_{B_{s}(x)}\left|y_{m}\right|^{\gamma q}|v-\bar{v}|^{q} \mathrm{~d} y\right)^{\frac{1}{q}} \\
& \cdot\left(\frac{1}{\left|B_{S}(x)\right|_{-\gamma \frac{q}{q-1}}} \int_{B_{S}(x)}\left|y_{m}\right|^{-\gamma \frac{q}{q-1}}|X|^{\frac{q}{q-1}} \mathrm{~d} y\right)^{\frac{q-1}{q}} \text {. }
\end{aligned}
$$


Note that since $\gamma$ satisfies (7.3), the right hand side of (7.5) is finite. Now we observe that

$$
\frac{\left|B_{S}(x)\right|_{\gamma q}^{\frac{1}{q}}\left|B_{S}(x)\right|_{-\gamma \frac{q}{q-1}}^{\frac{q-1}{q}}}{\left|B_{S}(x)\right|}=\left(\frac{\int_{B_{s}(x)}\left|y_{m}\right|^{\gamma q} \mathrm{~d} y}{\left|B_{S}(x)\right|}\left(\frac{\int_{B_{S}(x)}\left|y_{m}\right|^{-\gamma \frac{q}{q-1}} \mathrm{~d} y}{\left|B_{S}(x)\right|}\right)^{q-1}\right)^{\frac{1}{q}}
$$

which is precisely the quantity in the condition that $\left|y_{m}\right|^{\gamma q}$ is an $A_{q}(\mathrm{~d} y)$ weight (to the power of $\frac{1}{q}$ ). Since $\gamma$ satisfies (7.3), we have $\gamma \in\left(-\frac{1}{q}, 1-\frac{1}{q}\right)$ and Lemma 7.5 below implies that $\left|y_{m}\right|^{\gamma q}$ is an $A_{q}(\mathrm{~d} y)$ weight. Hence,

$$
\frac{\left|B_{S}(x)\right|_{\gamma q}^{\frac{1}{q}}\left|B_{S}(x)\right|_{-\gamma \frac{q}{q-1}}^{\frac{q-1}{q}}}{\left|B_{S}(x)\right|} \leq C(m, q, \gamma) .
$$

We apply Lemma 7.1 with $p=q \frac{m-1}{m}$ and $\kappa=\frac{m}{m-1}$ so that $\kappa p=q$, noting that our choice of $\gamma$ and Lemma 7.5 imply $\left|y_{m}\right|^{\gamma q}$ is an $A_{q \frac{m-1}{m}}(\mathrm{~d} y)$ weight. This yields

$$
\begin{aligned}
& \frac{1}{s}\left(\frac{1}{\left|B_{S}(x)\right|_{\gamma q}} \int_{B_{s}(x)}\left|y_{m}\right|^{\gamma q}|v-\bar{v}|^{q} \mathrm{~d} y\right)^{\frac{1}{q}} \\
& \leq C\left(\frac{1}{\left|B_{S}(x)\right|_{\gamma q}} \int_{B_{S}(x)}\left|y_{m}\right|^{\gamma q}|D v|^{\frac{q(m-1)}{m}} \mathrm{~d} y\right)^{\frac{m}{q(m-1)}} .
\end{aligned}
$$

We combine (7.4), (7.5), (7.7) and (7.8) to see that

$$
\begin{aligned}
& -s^{-m-1} \int_{B_{s}(x)} D \psi\left(\frac{x-y}{s}\right)(v-\bar{v}) \cdot X \mathrm{~d} y \\
& \leq C\left(M_{\gamma q}\left(|D v|^{\frac{q(m-1)}{m}}\right)(x)\right)^{\frac{m}{q(m-1)}}\left(M_{-\gamma \frac{q}{q-1}}\left(|X|^{\frac{q}{q-1}}\right)(x)\right)^{\frac{q-1}{q}},
\end{aligned}
$$

where we use the notation $M_{\gamma q}$ and $M_{-\gamma \frac{q}{q-1}}$ for the Maximal functions $M_{\mu_{1}}$ and $M_{\mu_{2}}$, as defined prior to Lemma 7.2, corresponding to the measures $\mathrm{d} \mu_{1}=\left|y_{m}\right|^{\gamma q} \mathrm{~d} y$ and $\mathrm{d} \mu_{2}=\left|y_{m}\right|^{-\gamma \frac{q}{q-1}} \mathrm{~d} y$, respectively. 
It follows from (7.9) and Hölder's inequality that

$$
\begin{aligned}
& \left\|\sup _{\psi \in \mathcal{F}} \sup _{s>0} \psi_{S} *(D v \cdot X)\right\|_{L^{1}\left(\mathbb{R}^{m}\right)} \\
& \quad \leq C \int_{\mathbb{R}^{m}}\left(M_{\gamma q}\left(|D v|^{\frac{q(m-1)}{m}}\right)\right)^{\frac{m}{q(m-1)}}\left(M_{-\gamma \frac{q}{q-1}}\left(|X|^{\frac{q}{q-1}}\right)\right)^{\frac{q-1}{q}} \mathrm{~d} x \\
& \quad=C \int_{\mathbb{R}^{m}}\left|x_{m}\right|^{\frac{\beta}{2}}\left(M_{\gamma q}\left(|D v|^{\frac{q(m-1)}{m}}\right)\right)^{\frac{m}{q(m-1)}}\left|x_{m}\right|^{-\frac{\beta}{2}}\left(M_{-\gamma \frac{q}{q-1}}\left(|X|^{\frac{q}{q-1}}\right)\right)^{\frac{q-1}{q}} \mathrm{~d} x \\
& \quad \leq C\left(\int_{\mathbb{R}^{m}}\left|x_{m}\right|^{\beta}\left(M_{\gamma q}\left(|D v|^{\frac{q(m-1)}{m}}\right)\right)^{2 \frac{m}{q(m-1)}} \mathrm{d} x\right)^{\frac{1}{2}} \\
& \quad\left(\int_{\mathbb{R}^{m}}\left|x_{m}\right|^{-\beta}\left(M_{-\gamma \frac{q}{q-1}}\left(|X|^{\frac{q}{q-1}}\right)\right)^{2 \frac{q-1}{q}} \mathrm{~d} x\right)^{\frac{1}{2}} .
\end{aligned}
$$

Now we apply Lemma 7.2 to two sets of measures. We write $\mathrm{d} \mu_{1}=\left|x_{m}\right|^{\gamma q} \mathrm{~d} y$ and $\mathrm{d} \nu_{1}=\left|x_{m}\right|^{\beta-\gamma q} \mathrm{~d} \mu_{1}=\left|x_{m}\right|^{\beta} \mathrm{d} y$. It follows from Lemma 7.5 that $\left|x_{m}\right|^{\beta-\gamma q}$ is an $A_{2 \frac{m}{q(m-1)}}\left(\mathrm{d} \mu_{1}\right)$ weight; hence, Lemma 7.2 yields

$$
\int_{\mathbb{R}^{m}}\left|x_{m}\right|^{\beta}\left(M_{\gamma q}\left(|D v|^{\frac{q(m-1)}{m}}\right)\right)^{2 \frac{m}{q(m-1)}} \mathrm{d} x \leq C \int_{\mathbb{R}^{m}}\left|x_{m}\right|^{\beta}|D v|^{2} \mathrm{~d} x .
$$

Similarly we write $\mathrm{d} \mu_{2}=\left|x_{m}\right|^{-\gamma \frac{q}{q-1}} \mathrm{~d} x$ and $\mathrm{d} \nu_{2}=\left|x_{m}\right|^{\gamma \frac{q}{q-1}-\beta} \mathrm{d} \mu_{2}=\left|x_{m}\right|^{-\beta} \mathrm{d} x$. It follows from Lemma 7.5 that $\left|x_{m}\right|^{\gamma \frac{q}{q-1}-\beta}$ is an $A_{2 \frac{q-1}{q}}\left(\mathrm{~d} \mu_{2}\right)$ weight, and hence, it follows from Lemma 7.2 that

$$
\int_{\mathbb{R}^{m}}\left|x_{m}\right|^{-\beta}\left(M_{-\gamma \frac{q}{q-1}}\left(|X|^{\frac{q}{q-1}}\right)\right)^{2 \frac{q-1}{q}} \mathrm{~d} x \leq C \int_{\mathbb{R}^{m}}\left|x_{m}\right|^{-\beta}|X|^{2} \mathrm{~d} x .
$$

The conclusion of the Lemma follows from (7.10), (7.11) and (7.12).

Next we prove a local version of Lemma 7.3.

Lemma 7.4 Let $\beta \in(-1,1), m \geq 3, v \in W_{\beta}^{1,2}\left(B_{r}\left(x_{0}\right) ; \mathbb{R}\right)$ and suppose that $X \in$ $L_{-\beta}^{2}\left(B_{r}\left(x_{0}\right) ; \mathbb{R}^{m}\right)$ with $\operatorname{div}(X)=0$ weakly in $B_{r}\left(x_{0}\right)$. Let $\eta \in C_{0}^{\infty}\left(B_{r}\left(x_{0}\right) ; \mathbb{R}\right)$ with $0 \leq \eta \leq 1, \eta=1$ in $B_{\frac{r}{2}}\left(x_{0}\right)$ and $|D \eta| \leq \frac{C_{0}}{r}$. Extend $D v$ and $X$ by 0 to $\mathbb{R}^{m}$ and let $\lambda=\left(\int_{\mathbb{R}^{m}} \eta \mathrm{d} x\right)^{-1} \int_{\mathbb{R}^{m}} \eta D v \cdot X \mathrm{~d} x$. Then $\eta(D v \cdot X-\lambda) \in \mathcal{H}^{1}\left(\mathbb{R}^{m}\right)$ and, for some constant $C=C\left(m, \beta, C_{0}\right)$ we have

$$
\|\eta(D v \cdot X-\lambda)\|_{\mathcal{H}^{1}\left(\mathbb{R}^{m}\right)} \leq C\|D v\|_{L_{\beta}^{2}\left(B_{r}\left(x_{0}\right) ; \mathbb{R}^{m}\right)}\|X\|_{L_{-\beta}^{2}\left(B_{r}\left(x_{0}\right) ; \mathbb{R}^{m}\right)}
$$

Remark The constant $\lambda$ is subtracted since it is a necessary condition that $\int_{\mathbb{R}^{m}} g \mathrm{~d} x=0$ for any $g \in \mathcal{H}^{1}\left(\mathbb{R}^{m}\right)$, see e.g. [28]. 
Proof Let $\psi \in C_{0}^{\infty}\left(B_{1}(0)\right)$ with $|D \psi| \leq 1$ and define $\psi_{s}(x)=s^{-m} \psi\left(\frac{x}{s}\right)$ for $s>0$. We have

$$
\psi_{s} *(\eta(D v \cdot X-\lambda))=\psi_{s} *(\eta D v \cdot X)-\lambda \psi_{s} * \eta .
$$

Let $\bar{v} \in \mathbb{R}$ denote a constant which will later be chosen to be the average $v$ over an appropriate set. Since $X$ is weakly divergence free in $B_{r}\left(x_{0}\right)$, we see that for any $x \in \mathbb{R}^{m}$ we have

$$
\begin{aligned}
\psi_{s} *(\eta D v \cdot X)(x)= & s^{-m} \int_{B_{s}(x) \cap B_{r}\left(x_{0}\right)} \psi\left(\frac{x-y}{s}\right) \eta D v \cdot X \mathrm{~d} y \\
= & s^{-m-1} \int_{B_{s}(x) \cap B_{r}\left(x_{0}\right)} D \psi\left(\frac{x-y}{s}\right) \eta(v-\bar{v}) \cdot X \mathrm{~d} y \\
& -s^{-m} \int_{B_{S}(x) \cap B_{r}\left(x_{0}\right)} \psi\left(\frac{x-y}{s}\right)(v-\bar{v}) D \eta \cdot X \mathrm{~d} y .
\end{aligned}
$$

For all $x \in \mathbb{R}^{m}, \bar{v} \in \mathbb{R}$ and $s>0$ we calculate

$$
\begin{aligned}
& \left|s^{-m-1} \int_{B_{s}(x) \cap B_{r}\left(x_{0}\right)} D \psi\left(\frac{x-y}{s}\right) \eta(v-\bar{v}) \cdot X \mathrm{~d} y\right| \\
& \leq \frac{C}{s\left|B_{S}(x)\right|} \int_{B_{S}(x) \cap B_{r}\left(x_{0}\right)}|v-\bar{v}||X| \mathrm{d} y .
\end{aligned}
$$

and, using that $|D \eta| \leq C_{0} r^{-1}$, we also find

$$
\begin{aligned}
& \left|s^{-m} \int_{B_{s}(x) \cap B_{r}\left(x_{0}\right)} \psi\left(\frac{x-y}{s}\right)(v-\bar{v}) D \eta \cdot X \mathrm{~d} y\right| \\
& \leq \frac{C}{\left|B_{S}(x)\right|} \int_{B_{s}(x) \cap B_{r}\left(x_{0}\right)}|v-\bar{v}||D \eta||X| \mathrm{d} y \\
& \quad \leq \frac{C}{r\left|B_{S}(x)\right|} \int_{B_{s}(x) \cap B_{r}\left(x_{0}\right)}|v-\bar{v}||X| \mathrm{d} y .
\end{aligned}
$$

We further observe that

$$
\left|\lambda \psi_{s} * \eta(x)\right|=\left|\lambda s^{-m} \int_{B_{s}(x) \cap B_{r}\left(x_{0}\right)} \psi\left(\frac{x-y}{s}\right) \eta \mathrm{d} y\right| \leq C|\lambda| s^{-m}\left|B_{s}(x) \cap B_{r}\left(x_{0}\right)\right| .
$$

We combine (7.13) - (7.16) to see that for all $x \in \mathbb{R}^{m}, \bar{v} \in \mathbb{R}$ and $s>0$ we have

$$
\left|\psi_{s} *(\eta(D v \cdot X-\lambda))(x)\right| \leq \frac{C}{\left|B_{S}(x)\right|}\left(s^{-1}+r^{-1}\right) \int_{B_{S}(x) \cap B_{r}\left(x_{0}\right)}|v-\bar{v}||X| \mathrm{d} y+C|\lambda| .
$$

To proceed we consider the cases $x \in B_{2 r}\left(x_{0}\right)$ and $x \in \mathbb{R}^{m} \backslash B_{2 r}\left(x_{0}\right)$ and recall that $\bar{v} \in \mathbb{R}$ is a constant we may choose. Suppose $x \in B_{2 r}\left(x_{0}\right)$ and let $s>0$. For 
$s \leq r$ we have $r^{-1} \leq s^{-1}$ and in this case we set $\bar{v}=\bar{v}_{B_{s}(x)}$ in (7.17). If $s>r$ then $B_{s}(x) \cap B_{r}\left(x_{0}\right) \subset B_{r}\left(x_{0}\right) \subset B_{3 r}(x)$ and we set $\bar{v}=\bar{v}_{B_{3 r}(x)}$ and observe that $\left|B_{S}(x)\right|^{-1} \leq 3^{m}\left|B_{3 r}(x)\right|^{-1}$. Then we see that for $x \in B_{2 r}\left(x_{0}\right)$ we have

$$
\left|\psi_{S} *(\eta(D v \cdot X-\lambda))(x)\right| \leq \frac{C}{t\left|B_{t}(x)\right|} \int_{B_{t}(x)}\left|v-\bar{v}_{B_{t}(x)}\right||X| \mathrm{d} y+C|\lambda|,
$$

where $t=s$ if $s \leq r$ and $t=3 r$ if $s>r$. Now, choose $q$ as in the proof of Lemma 7.3 and $\gamma$ as in (7.3). Noting that $X$ and $D v$ are extended by 0 outside $B_{r}\left(x_{0}\right)$ we conclude from (7.18) and (7.5)-(7.9) that

$$
\begin{aligned}
\left|\psi_{s} *(\eta(D v \cdot X-\lambda))(x)\right| \leq & C\left(M_{\gamma q}\left(|D v|^{\frac{q(m-1)}{m}}\right)(x)\right)^{\frac{m}{q(m-1)}}\left(M_{-\gamma \frac{q}{q-1}}\left(|X|^{\frac{q}{q-1}}\right)(x)\right)^{\frac{q-1}{q}} \\
& +C|\lambda|
\end{aligned}
$$

for every $x \in B_{2 r}\left(x_{0}\right)$ and $s>0$, where $M_{\gamma q}$ and $M_{-\gamma \frac{q}{q-1}}$ are the maximal functions as described in Lemma 7.3.

Now we consider $x \in \mathbb{R}^{m} \backslash B_{2 r}\left(x_{0}\right)$. Then $\left|x-x_{0}\right| \geq 2 r$. We observe that when $\left|x-x_{0}\right| \geq r+s$ we have $\psi_{s} *(\eta(D v \cdot X-\lambda))(x)=0$. Hence, we only need to consider the case when $r<s$ and $2 r \leq\left|x-x_{0}\right|<r+s$ (if $s \leq r$ this condition is empty). In this case we observe that $s^{-1}<2\left|x-x_{0}\right|^{-1}$. Hence, similarly to the proof of Proposition 1.92 of [28], we take advantage of the fact that $\int_{\mathbb{R}^{m}} \eta(D v \cdot X-\lambda) \mathrm{d} y=0$, the Mean Value Theorem and the fact that $|D \psi| \leq 1$ to see that

$$
\begin{aligned}
\left|\psi_{s} *(\eta(D v \cdot X-\lambda))(x)\right| & =s^{-m}\left|\int_{\mathbb{R}^{m}} \psi\left(\frac{x-y}{s}\right) \eta(D v \cdot X-\lambda) \mathrm{d} y\right| \\
& =s^{-m}\left|\int_{B_{r}\left(x_{0}\right)}\left(\psi\left(\frac{x-y}{s}\right)-\psi\left(\frac{x-x_{0}}{s}\right)\right) \eta(D v \cdot X-\lambda) \mathrm{d} y\right| \\
& \leq C s^{-m} s^{-1} \int_{B_{r}\left(x_{0}\right)}\left|x_{0}-y\right||D v \cdot X-\lambda| \mathrm{d} y \\
& \leq C r\left|x-x_{0}\right|^{-(m+1)} \int_{B_{r}\left(x_{0}\right)}|D v \cdot X|+|\lambda| \mathrm{d} y
\end{aligned}
$$

We apply Hölder's inequality to see that

$$
\begin{aligned}
& \left|\psi_{S} *(\eta(D v \cdot X-\lambda))(x)\right| \\
& \quad \leq C r\left|x-x_{0}\right|^{-(m+1)}\left(|| D v \|_{L_{\beta}^{2}\left(B_{r}\left(x_{0}\right) ; \mathbb{R}^{m}\right)}|| X||_{L_{-\beta}^{2}\left(B_{r}\left(x_{0}\right) ; \mathbb{R}^{m}\right)}+|\lambda| r^{m}\right),
\end{aligned}
$$


for every $x \in \mathbb{R}^{m} \backslash B_{2 r}\left(x_{0}\right)$ and every $s>0$. Now we estimate the $\mathcal{H}^{1}\left(\mathbb{R}^{m}\right)$ norm of $\eta(D v \cdot X-\lambda)$. We combine (7.19) and (7.21) to see that

$$
\begin{aligned}
\left\|\sup _{\psi \in \mathcal{F}} \sup _{s>0} \psi_{s} *(\eta(D v \cdot X-\lambda))\right\| & \\
= & \int_{B_{2 r}\left(x_{0}\right)}\left|\sup _{\psi \in \mathcal{F}} \sup _{s>0} \psi_{s} *(\eta(D v \cdot X-\lambda))\right| \mathrm{d} x \\
& +\int_{\mathbb{R}^{m} \backslash \mathbb{R}_{2 r}\left(x_{0}\right)}\left|\sup _{\psi \in \mathcal{F}} \sup _{s>0} \psi_{s} *(\eta(D v \cdot X-\lambda))\right| \mathrm{d} x \\
\leq & C \int_{B_{2 r}\left(x_{0}\right)}\left(M_{\gamma q}\left(|D v|^{\frac{q(m-1)}{m}}\right)(x)\right)^{\frac{m}{q(m-1)}}\left(M_{-\gamma} \frac{q}{q-1}\left(|X|^{\frac{q}{q-1}}\right)(x)\right)^{\frac{q-1}{q}}+|\lambda| \mathrm{d} x \\
& +C r \int_{\mathbb{R}^{m} \backslash B_{2 r}\left(x_{0}\right)}\left|x-x_{0}\right|^{-(m+1)}\left(\|D v\|_{L_{\beta}^{2}\left(B_{r}\left(x_{0}\right) ; \mathbb{R}^{m}\right)}|| X||_{L_{-\beta}^{2}\left(B_{r}\left(x_{0}\right) ; \mathbb{R}^{m}\right)}+|\lambda| r^{m}\right) \mathrm{d} x \\
\leq & C \int_{\mathbb{R}^{m}}\left(M_{\gamma q}\left(|D v|^{\frac{q(m-1)}{m}}\right)(x)\right)^{\frac{m}{q(m-1)}}\left(M_{-\gamma} \frac{q}{q-1}\left(|X|^{\frac{q}{q-1}}\right)(x)\right)^{\frac{q-1}{q}} \mathrm{~d} x+C r^{m}|\lambda| \\
& +C r\left(\left.\|D v\|\right|_{L_{\beta}^{2}\left(B_{r}\left(x_{0}\right) ; \mathbb{R}^{m}\right)}|| X \|_{L_{-\beta}^{2}\left(B_{r}\left(x_{0}\right) ; \mathbb{R}^{m}\right)}+|\lambda| r^{m}\right) \int_{\mathbb{R}^{m} \backslash B_{2 r}\left(x_{0}\right)}\left|x-x_{0}\right|^{-(m+1)} \mathrm{d} x .
\end{aligned}
$$

Now we observe that

$$
\int_{\mathbb{R}^{m} \backslash B_{2 r}\left(x_{0}\right)}\left|x-x_{0}\right|^{-(m+1)} \mathrm{d} x=2^{-1} r^{-1} \int_{\mathbb{R}^{m} \backslash B_{1}(0)}|x|^{-(m+1)} \mathrm{d} x=C r^{-1}
$$

and, using Hölder's inequality, that

$$
\begin{aligned}
& |\lambda| \leq C r^{-m} \int_{B_{r}\left(x_{0}\right)}\left|x_{m}\right|^{\frac{\beta}{2}}\left|x_{m}\right|^{-\frac{\beta}{2}}|D v \| X| \mathrm{d} x \\
& \leq C r^{-m}|| D v\left\|_{L_{\beta}^{2}\left(B_{r}\left(x_{0}\right) ; \mathbb{R}^{m}\right)}\right\| X \|_{L_{-\beta}^{2}\left(B_{r}\left(x_{0}\right) ; \mathbb{R}^{m}\right)} .
\end{aligned}
$$

We use (7.10) - (7.12) from the proof of Lemma 7.3, together with the fact that $D v$ and $X$ are extended by 0 in $\mathbb{R}^{m} \backslash B_{r}\left(x_{0}\right)$ to see that

$$
\begin{aligned}
& \int_{\mathbb{R}^{m}}\left(M_{\gamma q}\left(|D v|^{\frac{q(m-1)}{m}}\right)\right)^{\frac{m}{q(m-1)}}\left(M_{-\gamma \frac{q}{q-1}}\left(|X|^{\frac{q}{q-1}}\right)\right)^{\frac{q-1}{q}} \mathrm{~d} x \\
& \leq C\|D v\| L_{L^{2}\left(B_{r}\left(x_{0}\right) ; \mathbb{R}^{m}\right)}\|X\|_{L_{-\beta}^{2}\left(B_{r}\left(x_{0}\right) ; \mathbb{R}^{m}\right)} .
\end{aligned}
$$

We combine (7.22)-(7.25) to conclude the proof.

In the following lemma we give conditions for the measures discussed in the proof of Lemma 7.3 to satisfy their respective $A_{p}$ conditions.

Lemma 7.5 Let $m \geq 3, q \in\left(2, \frac{2 m}{m-1}\right)$ and $\beta \in(-1,1)$. Let $\mathrm{d} y$ be the Lebesgue measure on $\mathbb{R}^{m}$. Define $\mathrm{d} \mu_{1}=\left|y_{m}\right|^{\gamma q} \mathrm{~d} y$, and $\mathrm{d} \mu_{2}=\left|y_{m}\right|^{-\gamma \frac{q}{q-1}} \mathrm{~d} y$. Then 
1. for $\gamma \in\left(-\frac{1}{q}, 1-\frac{1}{q}\right)$ the weight $\left|y_{m}\right|^{\gamma q}$ is in $A_{q}(\mathrm{~d} y)$;

2. for $\gamma \in\left(-\frac{1}{q}, 1-\frac{m+q}{m q}\right)$ the weight $\left|y_{m}\right|^{\gamma q}$ is in $A_{q \frac{m-1}{m}}(\mathrm{~d} y)$;

3. for $\gamma>(\beta+1) \frac{m-1}{2 m}-\frac{1}{q}$, the weight $\left|y_{m}\right|^{\beta-\gamma q}$ is in $A_{2 \frac{m}{q(m-1)}}\left(\mathrm{d} \mu_{1}\right)$; and

4. for $\gamma<\min \left\{\frac{1+\beta}{2}, 1\right\}-\frac{1}{q}$, the weight $\left|y_{m}\right|^{\gamma \frac{q}{q-1}-\beta}$ is in $A_{2 \frac{q-1}{q}}\left(\mathrm{~d} \mu_{2}\right)$.

Proof To establish the Lemma, we show (7.1) is satisfied in each case. In order to do this we consider balls centred at $x=\left(x^{\prime}, x_{m}\right) \in \mathbb{R}^{m}$ with $\operatorname{dist}\left(B_{r}(x) ; \mathbb{R}^{m-1} \times\{0\}\right)=$ $\left|x_{m}\right|-r \geq r$ and balls with $0 \leq\left|x_{m}\right|<2 r$ separately. The subsequent calculations are similar for all four statements. We give the details for the first one and leave the rest to the reader, that is, we establish that $\left|y_{m}\right|^{\gamma q}$ is in $A_{q}(\mathrm{~d} y)$.

Suppose first that $\left|x_{m}\right|-r \geq r$. Then

$$
\frac{\left|x_{m}\right|}{2} \leq\left|y_{m}\right| \leq 2\left|x_{m}\right|
$$

for $y=\left(y^{\prime}, y_{m}\right) \in B_{r}(x)$. Hence,

$$
\begin{aligned}
\frac{1}{\left|B_{r}(x)\right|} \int_{B_{r}(x)}\left|y_{m}\right|^{\gamma q} \mathrm{~d} y\left(\frac{1}{\left|B_{r}(x)\right|} \int_{B_{r}(x)}\left|y_{m}\right|^{-\gamma \frac{q}{q-1}} \mathrm{~d} y\right)^{q-1} & \leq C\left|x_{m}\right|^{\gamma q}\left(\left|x_{m}\right|^{-\gamma \frac{q}{q-1}}\right)^{q-1} \\
& \leq C .
\end{aligned}
$$

Now suppose $0 \leq\left|x_{m}\right|<2 r$. Then $B_{r}(x) \subset B_{3 r}\left(x^{\prime}, 0\right)$. Provided $\gamma q>-1$ and $-\gamma \frac{q}{q-1}>-1$, we have

$$
\begin{aligned}
& \frac{1}{\left|B_{r}(x)\right|} \int_{B_{r}(x)}\left|y_{m}\right|^{\gamma q} \mathrm{~d} y\left(\frac{1}{\left|B_{r}(x)\right|} \int_{B_{r}(x)}\left|y_{m}\right|^{-\gamma \frac{q}{q-1}} \mathrm{~d} y\right)^{q-1} \\
& \quad \leq C r^{-m} \int_{B_{3 r}\left(x^{\prime}, 0\right)}\left|y_{m}\right|^{\gamma q} \mathrm{~d} y\left(r^{-m} \int_{B_{3 r}\left(x^{\prime}, 0\right)}\left|y_{m}\right|^{-\gamma \frac{q}{q-1}} \mathrm{~d} y\right)^{q-1} \\
& \quad \leq C r^{-q} \int_{0}^{3 r} t^{\gamma q} \mathrm{~d} t\left(\int_{0}^{3 r} t^{-\gamma \frac{q}{q-1}} \mathrm{~d} t\right)^{q-1} \leq C .
\end{aligned}
$$

Hence, we have verified (7.1).

\section{Caccioppoli-Type Inequality and Energy Decay}

With the Weighted Hardy estimate in Lemma 7.3 in hand, we may prove a CaccioppoliType inequality for stationary harmonic maps with respect to free boundary data. This estimate gives control of the re-scaled energy on a half-ball in terms of the mean squared oscillation and re-scaled energy on a half-ball of twice the radius. We will then use this lemma to show the re-scaled energy decays faster than implied by the monotonicity formula stated in Sect. 4; we will show the decay we obtain is fast enough to imply Hölder continuity in Sect. 9. 
We need the following preliminary lemma with regard to extending a $B M O\left(B_{r}\left(x_{0}\right)\right)$ function to $B M O\left(\mathbb{R}^{m}\right)$ by multiplying by a cutoff function. The following lemma follows from well-known arguments, we give a proof based on e.g [6] Lemma 4.1 in order to elucidate the dependence of the constants on the cutoff function.

Lemma 8.1 Suppose $v \in B M O\left(B_{r}\left(x^{*}\right)\right)$ for some ball $B_{r}\left(x^{*}\right) \subset \mathbb{R}^{m}$. Let $\eta \in$ $C_{0}^{\infty}\left(B_{\frac{3 r}{4}}\left(x^{*}\right)\right)$ with $\eta \equiv 1$ in $B_{\frac{r}{2}}\left(x^{*}\right), 0 \leq \eta \leq 1$ and $|D \eta| \leq C^{*} r^{-1}$. Then $w=\eta\left(v-\bar{v}_{B_{r}\left(x^{*}\right)}\right) \in B M O\left(\mathbb{R}^{m}\right)$ and $[w]_{B M O\left(\mathbb{R}^{m}\right)} \leq C[v]_{B M O\left(B_{r}\left(x^{*}\right)\right)}$ for a constant $C=C(m)\left(1+C^{*}\right)$.

Proof We assume with no loss of generality that $\bar{v}_{B_{r}\left(x^{*}\right)}=0$.

Observe that

$$
\frac{1}{\left|B_{S}(x)\right|} \int_{B_{S}(x)}\left|w-\bar{w}_{B_{S}(x)}\right| \mathrm{d} y \leq \frac{2}{\left|B_{S}(x)\right|} \int_{B_{s}(x) \cap B_{r}\left(x^{*}\right)}|w| \mathrm{d} y .
$$

If $s \geq \frac{r}{16 \sqrt{m}}$ then it follows from (8.1) that

$$
\begin{aligned}
\frac{1}{\left|B_{S}(x)\right|} \int_{B_{S}(x)}\left|w-\bar{w}_{B_{s}(x)}\right| \mathrm{d} y & \leq \frac{2}{\left|B_{S}(x)\right|} \int_{B_{s}(x) \cap B_{r}\left(x^{*}\right)}|w| \mathrm{d} y \\
& \leq \frac{C}{\left|B_{r}\left(x^{*}\right)\right|} \int_{B_{r}\left(x^{*}\right)}|v| \mathrm{d} y \\
& \leq C[v]_{B M O\left(B_{r}\left(x^{*}\right)\right)}
\end{aligned}
$$

To proceed, we consider the cases $x \in B_{\frac{13}{16}} r\left(x^{*}\right)$ and $x \in \mathbb{R}^{m} \backslash B_{\frac{13}{16}} r\left(x^{*}\right)$. First suppose $x \in \mathbb{R}^{m} \backslash B_{\frac{13}{16} r}\left(x^{*}\right)$. If $s \geq \frac{r}{16 \sqrt{m}}$ then (8.2) holds and if $s<\frac{r}{16 \sqrt{m}}$ then $B_{S}(x) \cap B_{\frac{3 r}{4}}\left(x^{*}\right)=\emptyset$ and it follows from (8.1) and the fact that $\eta \in C_{0}^{\infty}\left(B_{\frac{3 r}{4}}\left(x^{*}\right) ; \mathbb{R}\right)$ that

$$
\frac{1}{\left|B_{S}(x)\right|} \int_{B_{S}(x)}\left|w-\bar{w}_{B_{s}(x)}\right| \mathrm{d} y=0
$$

Now suppose $x \in B_{\frac{13}{16}}\left(x^{*}\right)$. If $s \geq \frac{r}{16 \sqrt{m}}$ then (8.2) still holds. Hence, we consider $0 \leq s<\frac{r}{16 \sqrt{m}}$ and note that in this case $B_{s}(x) \subset B_{\frac{7}{8} r}\left(x^{*}\right)$. Observe that

$$
w-\bar{w}_{B_{S}(x)}=\eta v-\overline{\eta v}_{B_{s}(x)}=\eta\left(v-\bar{v}_{B_{s}(x)}\right)+\left|B_{S}(x)\right|^{-1} \int_{B_{s}(x)} v(y)(\eta(z)-\eta(y)) \mathrm{d} y .
$$


Consequently, using the fact that $|D \eta| \leq C^{*} r^{-1}$, we see that

$$
\begin{aligned}
& \frac{1}{\left|B_{S}(x)\right|} \int_{B_{S}(x)}\left|w-\bar{w}_{B_{S}(x)}\right| \mathrm{d} z \\
& \quad \leq[v]_{B M O\left(B_{r}\left(x^{*}\right)\right)}+\left.\frac{1}{\left|B_{S}(x)\right|} \int_{B_{S}(x)}|| B_{S}(x)\right|^{-1} \int_{B_{S}(x)} v(y)(\eta(z)-\eta(y)) \mathrm{d} y \mid \mathrm{d} z \\
& \quad \leq[v]_{B M O\left(B_{r}\left(x^{*}\right)\right)}+\frac{2 C^{*} s}{r\left|B_{S}(x)\right|} \int_{B_{S}(x)}|v| \mathrm{d} y
\end{aligned}
$$

Furthermore, we can use Hölder's inequality to estimate

$$
\left|B_{S}(x)\right|^{-1} \int_{B_{S}(x)}|v| \mathrm{d} y \leq\left(\left|B_{S}(x)\right|^{-1} \int_{B_{7 r / 8}\left(x^{*}\right)}|v|^{m} \mathrm{~d} y\right)^{\frac{1}{m}} .
$$

It follows from the John-Nirenberg inequality, see [8] Corollary 6.22, that

$$
\left(\int_{B_{7 r / 8}\left(x^{*}\right)}|v|^{m} \mathrm{~d} y\right)^{\frac{1}{m}} \leq C r[v]_{B M O\left(B_{r}\left(x^{*}\right)\right)}
$$

Together with (8.4) and (8.5), this yields the desired estimate.

Now we prove our Caccioppoli-type estimate.

Lemma 8.2 There exists a constant $C>0$ such that the following holds. Suppose $v \in W_{\beta}^{1,2}\left(\mathscr{U} ; \mathbb{S}^{n-1}\right)$ is weakly stationary harmonic with respect to free boundary data. Let $\tilde{v} \in W_{\beta}^{1,2}\left(\mathscr{V} ; \mathbb{S}^{n-1}\right)$ denote the even reflection of $v$ in $\mathbb{R}^{m-1} \times\{0\}$. Then for $B_{r}^{+}\left(x_{0}\right) \subset \mathscr{U}$ with $\left(x_{0}\right)_{m}=0, r>0$ and $B_{2 r}^{+}\left(x_{0}\right) \subset \mathscr{U}$ and any $\delta_{0}>0$ we have

$$
\begin{aligned}
\left(\frac{r}{2}\right)^{2-m-\beta} \int_{B_{\frac{r}{2}}^{+}\left(x_{0}\right)} x_{m}^{\beta}|D v|^{2} \mathrm{~d} x \leq & \left(C[\tilde{v}]_{B M O\left(B_{r}\left(x_{0}\right)\right)}+\delta_{0}\right) r^{2-m-\beta} \int_{B_{r}^{+}\left(x_{0}\right)} x_{m}^{\beta}|D v|^{2} \mathrm{~d} x \\
& +C \delta_{0}^{-1} r^{-(m+\beta)} \int_{B_{r}^{+}\left(x_{0}\right)} x_{m}^{\beta}\left|v-\bar{v}_{B_{r}^{+}\left(x_{0}\right), \beta}\right|^{2} \mathrm{~d} x
\end{aligned}
$$

Proof The proof is analogous to the case for stationary harmonic maps, see for instance Lemma 3.8 of [18]. Let $\eta \in C_{0}^{\infty}\left(B_{\frac{3}{4} r}\left(x_{0}\right) ; \mathbb{R}\right)$ be a smooth cutoff function with $\eta \equiv 1$ in $B_{\frac{r}{2}}\left(x_{0}\right), 0 \leq \eta \leq 1$ and $|D \eta| \leq \frac{C}{r}$. Henceforth, we abbreviate the notation $\overline{\tilde{v}}_{B_{r}\left(x_{0}\right), \beta}$ to $\overline{\tilde{v}}$ and $\bar{v}_{B_{r}^{+}\left(x_{0}\right), \beta}$ to $\bar{v}$. Let $\tilde{g}$ be the reflection of $g$. It follows from (2.4) and the fact that $|\tilde{v}|^{2}=1$ almost everywhere that

$$
\tilde{g}^{i j}\left\langle\partial_{i} \tilde{v}, \partial_{j} \tilde{v}\right\rangle=\frac{1}{2} \tilde{g}^{i j}\left(\tilde{v}^{a} \partial_{i} \tilde{v}^{b}-\tilde{v}^{b} \partial_{i} \tilde{v}^{a}\right)\left(\tilde{v}^{a} \partial_{j} \tilde{v}^{b}-\tilde{v}^{b} \partial_{j} \tilde{v}^{a}\right)
$$


Now let $w=\eta(\tilde{v}-\overline{\tilde{v}}) \in W_{\beta, 0}^{1,2}\left(B_{r}\left(x_{0}\right) ; \mathbb{R}^{n}\right)$. We calculate

$$
\begin{aligned}
\partial_{i}\left(\tilde{v}^{a} w^{b}-\tilde{v}^{b} w^{a}\right)= & \partial_{i} \eta\left(\tilde{v}^{a}(\tilde{v}-\overline{\tilde{v}})^{b}-(\tilde{v}-\overline{\tilde{v}})^{a} \tilde{v}^{b}\right) \\
& +\eta\left(\tilde{v}^{a} \partial_{i} \tilde{v}^{b}-\tilde{v}^{b} \partial_{i} \tilde{v}^{a}\right) \\
& +\partial_{i} \tilde{v}^{a} w^{b}-\partial_{i} \tilde{v}^{b} w^{a}
\end{aligned}
$$

for $a, b=1, \ldots, n$. We observe that since $\tilde{v}, w \in L^{\infty}\left(\mathscr{V} ; \mathbb{R}^{n}\right)$ it follows that $\tilde{v}^{a} w^{b}-$ $\tilde{v}^{b} w^{a} \in W_{\beta}^{1,2}\left(B_{r}\left(x_{0}\right) ; \mathbb{R}\right)$. Moreover, since the support of $\eta$ is a compact subset of $B_{r}\left(x_{0}\right)$, we see that $\tilde{v}^{a} w^{b}-\tilde{v}^{b} w^{a} \in W_{\beta, 0}^{1,2}\left(B_{r}\left(x_{0}\right) ; \mathbb{R}\right)$. We observe that the preceding discussion for $w$ also holds true for $W=\eta w$, including (8.8) which holds replacing $\eta$ with $\eta^{2}, \partial_{i} \eta$ with $2 \eta \partial_{i} \eta$ and $w$ with $W$.

Lemma 6.1 implies that the vector fields $\tilde{X}_{a b} \in L_{-\beta}^{2}\left(\mathscr{V} ; \mathbb{R}^{n}\right)$ given in components by $\tilde{X}_{a b}^{i}=\left|x_{m}\right|^{\beta} \sqrt{\operatorname{det}\left(g\left(x^{\prime},\left|x_{m}\right|\right)\right)} \tilde{g}^{i j}\left(\tilde{v}^{a} \partial_{j} \tilde{v}^{b}-\tilde{v}^{b} \partial_{j} \tilde{v}^{a}\right)$ are weakly divergence free in $\mathscr{V}$. We use this fact, together with the fact that $\tilde{v}^{a} W^{b}-\tilde{v}^{b} W^{a} \in W_{\beta, 0}^{1,2}\left(B_{r}\left(x_{0}\right) ; \mathbb{R}\right)$ and (8.7) and (8.8), to see that

$$
\begin{aligned}
& \int_{B_{\frac{r}{2}}\left(x_{0}\right)}\left|x_{m}\right|^{\beta} \tilde{g}^{i j}\left\langle\partial_{i} \tilde{v}, \partial_{j} \tilde{v}\right\rangle \sqrt{\operatorname{det}\left(g\left(x^{\prime},\left|x_{m}\right|\right)\right)} \mathrm{d} x \\
& \leq \frac{1}{2} \int_{B_{r}\left(x_{0}\right)} \eta^{2}\left|x_{m}\right|^{\beta} \tilde{g}^{i j}\left(\tilde{v}^{a} \partial_{i} \tilde{v}^{b}-\tilde{v}^{b} \partial_{i} \tilde{v}^{a}\right)\left(\tilde{v}^{a} \partial_{j} \tilde{v}^{b}-\tilde{v}^{b} \partial_{j} \tilde{v}^{a}\right) \sqrt{\operatorname{det}\left(g\left(x^{\prime},\left|x_{m}\right|\right)\right)} \mathrm{d} x \\
& =\frac{1}{2} \int_{B_{r}\left(x_{0}\right)} \eta^{2}\left(\tilde{v}^{a} \partial_{i} \tilde{v}^{b}-\tilde{v}^{b} \partial_{i} \tilde{v}^{a}\right) \tilde{X}_{a b}^{i} \mathrm{~d} x \\
& =-\int_{B_{r}\left(x_{0}\right)} \partial_{i} \eta\left(\tilde{v}^{a}(\tilde{v}-\overline{\tilde{v}})^{b}-(\tilde{v}-\overline{\tilde{v}})^{a} \tilde{v}^{b}\right) \eta \tilde{X}_{a b}^{i} \mathrm{~d} x \\
& \quad-\frac{1}{2} \int_{B_{r}\left(x_{0}\right)}\left(\partial_{i} \tilde{v}^{a} w^{b}-\partial_{i} \tilde{v}^{b} w^{a}\right) \eta \tilde{X}_{a b}^{i} \mathrm{~d} x .
\end{aligned}
$$

We apply Young's inequality to see that

$$
\begin{aligned}
& -\int_{B_{r}\left(x_{0}\right)} \partial_{i} \eta\left(\tilde{v}^{a}(\tilde{v}-\overline{\tilde{v}})^{b}-(\tilde{v}-\overline{\tilde{v}})^{a} \tilde{v}^{b}\right) \eta \tilde{X}_{a b}^{i} \mathrm{~d} x \\
& \leq C \int_{B_{r}\left(x_{0}\right)}\left|x_{m}\right|^{\beta}|D \eta||\tilde{v}-\overline{\tilde{v}}||D \tilde{v}| \mathrm{d} x \\
& \quad \leq \delta_{0} \int_{B_{r}^{+}\left(x_{0}\right)} x_{m}^{\beta}|D v|^{2} \mathrm{~d} x+\frac{C}{\delta_{0} r^{2}} \int_{B_{r}^{+}\left(x_{0}\right)} x_{m}^{\beta}|v-\bar{v}|^{2} \mathrm{~d} x
\end{aligned}
$$

where we have also used the fact that

$$
\int_{B_{r}\left(x_{0}\right)}\left|x_{m}\right|^{\beta}|\tilde{v}-\overline{\tilde{v}}|^{2} \mathrm{~d} x \leq C \int_{B_{r}^{+}\left(x_{0}\right)} x_{m}^{\beta}|v-\bar{v}|^{2} \mathrm{~d} x .
$$


It follows from Lemma 8.1 that

$$
w^{a} \in B M O\left(\mathbb{R}^{m}\right) \text { and }\left[w^{a}\right]_{B M O\left(\mathbb{R}^{m}\right)} \leq C_{0}\left[\tilde{v}^{a}\right]_{B M O\left(B_{r}\left(x_{0}\right)\right)}
$$

for $a=1, \ldots, m$. We now apply Lemma 7.4 which implies that for each $a, b=$ $1, \ldots, n$ we have $\eta\left(D \tilde{v}^{a} \cdot \tilde{X}_{a b}-\lambda_{a b}\right) \in \mathcal{H}^{1}\left(\mathbb{R}^{m}\right)$ where we have extended $\tilde{X}$ and $D \tilde{v}$ by 0 to $\mathbb{R}^{m}$ and $\lambda_{a b}=\left(\int_{\mathbb{R}^{m}} \eta \mathrm{d} x\right)^{-1} \int_{\mathbb{R}^{m}} \eta D v^{a} \cdot \tilde{X}_{a b} \mathrm{~d} x$. We further note that $\eta\left(D \tilde{v}^{a} \cdot \tilde{X}_{a b}-\lambda_{a b}\right) \in L^{1}\left(\mathbb{R}^{m}\right)$ and $w^{a} \in L^{\infty}\left(\mathbb{R}^{m}\right)$ for $a, b=1, \ldots, m$. We may therefore apply the duality of $\mathcal{H}^{1}\left(\mathbb{R}^{m}\right)$ and $B M O\left(\mathbb{R}^{m}\right)$, see [18] Lemma 2.3, together with Theorem 7.4 and the fact that $\tilde{X}_{a b}^{i}=-\tilde{X}_{b a}^{i}$, to see that

$$
\begin{aligned}
- & \int_{B_{r}\left(x_{0}\right)}\left(\partial_{i} \tilde{v}^{a} w^{b}-\partial_{i} \tilde{v}^{b} w^{a}\right) \eta \tilde{X}_{a b}^{i} \mathrm{~d} x \\
= & -2 \int_{B_{r}\left(x_{0}\right)} w^{b} D \tilde{v}^{a} \cdot \eta \tilde{X}_{a b} \mathrm{~d} x \\
= & -2 \int_{B_{r}\left(x_{0}\right)} w^{b} \eta\left(D \tilde{v}^{a} \cdot \tilde{X}_{a b}-\lambda_{a b}\right) \mathrm{d} x-2 \int_{B_{r}\left(x_{0}\right)} w^{b} \eta \lambda_{a b} \mathrm{~d} x \\
\leq & 2\left[w^{b}\right]_{B M O\left(\mathbb{R}^{m}\right)}|| \eta\left(D \tilde{v}^{a} \cdot \tilde{X}_{a b}-\lambda_{a b}\right)||_{\mathcal{H}^{1}\left(\mathbb{R}^{m}\right)}+2\left|\lambda_{a b}\right| \int_{B_{r}\left(x_{0}\right)}\left|\tilde{v}^{b}-\overline{\tilde{v}^{b}}\right| \mathrm{d} x \\
\leq & C\left[w^{b}\right]_{B M O\left(\mathbb{R}^{m}\right)}|| D \tilde{v}^{a}\left\|\left.\right|_{L_{\beta}^{2}\left(B_{r}\left(x_{0}\right) ; \mathbb{R}^{m}\right)}\right\| \tilde{X}_{a b} \|_{L_{-\beta}^{2}\left(B_{r}\left(x_{0}\right) ; \mathbb{R}^{m}\right)} \\
& +\left.C\left\|D \tilde{v}^{a}||_{L_{\beta}^{2}\left(B_{r}\left(x_{0}\right) ; \mathbb{R}^{m}\right)}|| \tilde{X}_{a b}\right\|\right|_{L_{-\beta}^{2}\left(B_{r}\left(x_{0}\right) ; \mathbb{R}^{m}\right)} \frac{1}{\left|B_{r}\left(x_{0}\right)\right|} \int_{B_{r}\left(x_{0}\right)}\left|\tilde{v}^{b}-\overline{\tilde{v}^{b}}\right| \mathrm{d} x \\
\leq & C\left[\tilde{v}^{b}\right]_{B M O\left(B_{r}\left(x_{0}\right)\right)}|| D \tilde{v}^{a}\left\|_{L_{\beta}^{2}\left(B_{r}\left(x_{0}\right) ; \mathbb{R}^{m}\right)}\right\| \tilde{X}_{a b} \|_{L_{-\beta}^{2}\left(B_{r}\left(x_{0}\right) ; \mathbb{R}^{m}\right)} \\
\leq & C[\tilde{v}]_{\left.B M O\left(B_{r}\left(x_{0}\right)\right)\right)} \int_{B_{r}^{+}\left(x_{0}\right)} x_{m}^{\beta}|D v|^{2} \mathrm{~d} x .
\end{aligned}
$$

Together (8.9), (8.10) and (8.11) yield (8.6) as required.

We now combine the Caccioppoli-type inequality in Lemma 8.2 with the control of the mean squared oscillation given by Lemma 5.2 to see that the re-scaled energy of free boundary stationary harmonic maps decays faster than implied by the energy monotonicity formula.

Lemma 8.3 There exist constants $R_{1}>0, \varepsilon_{1}>0$ and $\theta_{1} \in\left(0, \frac{1}{4}\right)$ such that the following holds. Suppose $v \in W_{\beta}^{1,2}\left(\mathscr{U} ; \mathbb{S}^{n-1}\right)$ is stationary harmonic with respect to free boundary data and consider a half-ball $B_{R}^{+}\left(x_{0}\right)$ with $R \leq R_{1}$ and $B_{2 R}^{+}\left(x_{0}\right) \subset \mathscr{U}$. If

$$
R^{2-m-\beta} \int_{B_{R}^{+}\left(x_{0}\right)} x_{m}^{\beta}|D v|_{g}^{2} \mathrm{dvol}_{g} \leq \varepsilon_{1},
$$


then for every $B_{\rho}^{+}(y) \subset B_{R}^{+}\left(x_{0}\right)$ with $y \in B_{\frac{R}{2}}\left(x_{0}\right) \cap\left(\mathbb{R}^{m-1} \times\{0\}\right)$ and $\rho \leq \frac{R}{2}$ we have

$$
\left(\theta_{1} \rho\right)^{2-m-\beta} \int_{B_{\theta_{1} \rho}^{+}(y)} x_{m}^{\beta}|D v|^{2} \mathrm{~d} x \leq \frac{1}{2} \rho^{2-m-\beta} \int_{B_{\rho}^{+}(y)} x_{m}^{\beta}|D v|^{2} \mathrm{~d} x .
$$

Proof Suppose $v$ satisfies $R^{2-m-\beta} \int_{B_{R}^{+}\left(x_{0}\right)} x_{m}^{\beta}|D v|_{g}^{2} \mathrm{dvol}_{g} \leq \varepsilon_{1}$ for $\varepsilon_{1}>0$ to be chosen. Corollary 4.2 then implies that for any $\rho \in\left(0, \frac{R}{2}\right]$ and $y \in B_{\frac{R}{2}}\left(x_{0}\right) \cap\left(\mathbb{R}^{m-1} \times\right.$ $\{0\})$ we have

$$
\begin{aligned}
\rho^{2-m-\beta} \int_{B_{\rho}^{+}(y)} x_{m}^{\beta}|D v|_{g}^{2} \mathrm{dvol}_{g} & \leq C\left(\frac{R}{2}\right)^{2-m-\beta} \int_{B_{\frac{R}{2}}^{+}(y)} x_{m}^{\beta}|D v|_{g}^{2} \mathrm{dvol}_{g} \\
& \leq C R^{2-m-\beta} \int_{B_{R}^{+}\left(x_{0}\right)} x_{m}^{\beta}|D v|_{g}^{2} \mathrm{dvol}_{g} \\
& \leq C \varepsilon_{1} .
\end{aligned}
$$

Since $v$ satisfies (6.1) and $|v|=1$ almost everywhere it satisfies (5.5) with $C^{*}=1$ for every $\psi \in C_{0}^{\infty}\left(\tilde{\mathscr{U}}, \mathbb{R}^{n}\right)$. Then for every $\delta>0$ there exist constants $\varepsilon_{0}=\varepsilon_{0}(\delta)>0$, $\theta_{0}=\theta_{0}(\delta) \in\left(0, \frac{1}{8}\right]$ and $R_{0}=R_{0}(\delta) \in(0,1]$ such that the conclusion of Lemma 5.2 holds. These numbers also may also depend on $m, \beta$ and other uniform constants but only the dependence on $\delta$ is important here. We, henceforth, assume $R_{1} \leq R_{0}$ and $\varepsilon_{1} \leq C^{-1} \varepsilon_{0}$ where $C$ is the constant from (8.13); we will subsequently fix $\delta$ uniformly. In view of (8.13) and the choice of $\varepsilon_{1}$ we have $\rho^{2-m-\beta} \int_{B_{\rho}^{+}(y)} x_{m}^{\beta}|D v|_{g}^{2} \mathrm{dvol}_{g} \leq \varepsilon_{0}$, and hence, it follows from Lemma 5.2 that

$$
\left(\theta_{0} \rho\right)^{-(m+\beta)} \int_{B_{\theta_{0} \rho}^{+}(y)} x_{m}^{\beta}\left|v-\bar{v}_{B_{\theta_{0} \rho}^{+}(y), \beta}\right|^{2} \mathrm{~d} x \leq \delta \rho^{2-m-\beta} \int_{B_{\rho}^{+}(y)} x_{m}^{\beta}|D v|_{g}^{2} \mathrm{dvol}_{g} .
$$


Let $\tilde{v} \in W_{\beta}^{1,2}\left(\mathscr{V} ; \mathbb{S}^{n-1}\right)$ denote the even reflection of $v$ in $\mathbb{R}^{m-1} \times\{0\}$. We apply Lemma 8.2 followed by Lemma 5.2, together with Corollary 4.2 to see that

$$
\begin{aligned}
& \left(\frac{\theta_{0} \rho}{2}\right)^{2-m-\beta} \int_{B_{\frac{\theta_{0} \rho}{2}}^{+}(y)} x_{m}^{\beta}|D v|^{2} \mathrm{~d} x \\
& \leq C\left([\tilde{v}]_{B M O\left(B_{\theta_{0} \rho}(y)\right)}+\delta_{0}\right)\left(\theta_{0} \rho\right)^{2-m-\beta} \int_{B_{\theta_{0} \rho}^{+}(y)} x_{m}^{\beta}|D v|^{2} \mathrm{~d} x \\
& \quad+C \delta_{0}^{-1}\left(\theta_{0} \rho\right)^{-(m+\beta)} \int_{B_{\theta_{0} \rho}^{+}(y)} x_{m}^{\beta}\left|v-\bar{v}_{B_{r}(y), \beta}\right|^{2} \mathrm{~d} x \\
& \leq C\left([\tilde{v}]_{B M O\left(B_{\theta_{0} \rho}(y)\right)}+\delta_{0}\right) \rho^{2-m-\beta} \int_{B_{\rho}^{+}(y)} x_{m}^{\beta}|D v|^{2} \mathrm{~d} x \\
& \quad+C \delta_{0}^{-1} \delta \rho^{2-m-\beta} \int_{B_{\rho}^{+}(y)} x_{m}^{\beta}|D v|^{2} \mathrm{~d} x \\
& =C\left([\tilde{v}]_{B M O\left(B_{\theta_{0} \rho}(y)\right)}+\delta_{0}+\delta \delta_{0}^{-1}\right) \rho^{2-m-\beta} \int_{B_{\rho}^{+}(y)} x_{m}^{\beta}|D v|^{2} \mathrm{~d} x .
\end{aligned}
$$

Now first choose $\delta_{0}=\frac{1}{8 C}$ and then choose $\delta=\frac{1}{64 C^{2}}$ where $C$ is the constant from (8.14). This fixes $\varepsilon_{1}$ and $\theta_{1}:=\frac{\theta_{0}}{2} \in\left(0, \frac{1}{8}\right]$ uniformly. It, hence, follows from (8.14) that

$$
\left(\theta_{1} \rho\right)^{2-m-\beta} \int_{B_{\theta_{1} \rho}^{+}(y)} x_{m}^{\beta}|D v|^{2} \mathrm{~d} x \leq\left(C[\tilde{v}]_{B M O\left(B_{\theta_{0} \rho}(y)\right)}+\frac{1}{4}\right) \rho^{2-m-\beta} \int_{B_{\rho}^{+}(y)} x_{m}^{\beta}|D v|^{2} \mathrm{~d} x
$$

Since $\theta_{0} \rho \leq \frac{\rho}{8}$, we may apply Lemma 4.2 to see that

$$
[\tilde{v}]_{B M O\left(B_{\theta_{0} \rho}(y)\right)} \leq C\left(\left(2 \theta_{0} \rho\right)^{2-m-\beta} \int_{B_{2 \theta_{0} \rho}^{+}(y)} x_{m}^{\beta}|D v|^{2} \mathrm{~d} x\right)^{\frac{1}{2}}
$$

We combine (8.16) with (8.15), (8.13) and the monotonicity formula from Corollary 4.2 to see that

$$
\left(\theta_{1} \rho\right)^{2-m-\beta} \int_{B_{\theta_{1} \rho}^{+}(y)} x_{m}^{\beta}|D v|^{2} \mathrm{~d} x \leq\left(\tilde{C} \varepsilon_{1}^{\frac{1}{2}}+\frac{1}{4}\right) \rho^{2-m-\beta} \int_{B_{\rho}^{+}(y)} x_{m}^{\beta}|D v|^{2} \mathrm{~d} x .
$$

To conclude the proof we fix $\varepsilon_{1}=\min \left\{\frac{1}{16 \tilde{C}^{2}}, C^{-1} \varepsilon_{0}\right\}$ where $C$ is the constant from (8.13). 


\section{$9 \varepsilon$-Regularity Near the Boundary and Partial Regularity}

With the results of the previous sections in hand, we are now in a position to prove $\varepsilon$-regularity for stationary harmonic maps with free boundary data near the boundary.

Theorem 9.1 There exists $\varepsilon_{2}>0, R_{2}>0$ and $\gamma, \theta_{2} \in(0,1)$ such that the following holds. Suppose $v \in W_{\beta}^{1,2}\left(\mathscr{U} ; \mathbb{S}^{n-1}\right)$ is a weakly stationary harmonic map with respect to free boundary data. If $B_{R}^{+}\left(x_{0}\right)$ is a half-ball with $R \leq R_{2}, B_{2 R}^{+}\left(x_{0}\right) \subset \mathscr{U}$ and

$$
R^{2-m-\beta} \int_{B_{R}^{+}\left(x_{0}\right)} x_{m}^{\beta}|D v|_{g}^{2} \operatorname{dvol}_{g} \leq \varepsilon_{2}
$$

then $v \in C^{0, \gamma}\left(\overline{B_{\theta_{2} R}^{+}\left(x_{0}\right)} ; \mathbb{S}^{n-1}\right)$.

Proof Our goal is to apply a version of Morrey's Decay Lemma, see for example [18] Lemma 2.1 for a precise statement. Let $\varepsilon_{1}>0, R_{1}>0$ and $\theta_{1} \in\left(0, \frac{1}{8}\right]$ be the numbers from Lemma 8.3. Consider $B_{\rho}^{+}(y)$ with $\rho \in\left(0, \frac{R}{2}\right]$ and $y \in B_{\frac{R}{2}}\left(x_{0}\right) \cap\left(\mathbb{R}^{m-1} \times\{0\}\right)$. If $\varepsilon_{2} \leq \varepsilon_{1}$ then the lemma applies and iterating (8.12) for $k \in \mathbb{N} \cup\{0\}$ yields

$$
\left(\theta_{1}^{k} \rho\right)^{2-m-\beta} \int_{B_{\theta_{1}^{k} \rho}^{+}(y)} x_{m}^{\beta}|D v|^{2} \mathrm{~d} x \leq \frac{1}{2^{k}} \rho^{2-m-\beta} \int_{B_{\rho}^{+}(y)} x_{m}^{\beta}|D v|^{2} \mathrm{~d} x .
$$

Now set $\gamma_{0}=-\ln (2)\left(\ln \left(\theta_{1}\right)\right)^{-1} \in(0,1)$. Then we have

$$
\left(\theta_{1}^{k} \rho\right)^{2-m-\beta} \int_{B_{\theta_{1}^{+} \rho}^{+}(y)} x_{m}^{\beta}|D v|^{2} \mathrm{~d} x \leq \frac{\left(\theta_{1}^{k} \rho\right)^{\gamma_{0}}}{\rho^{\gamma_{0}}} \rho^{2-m-\beta} \int_{B_{\rho}^{+}(y)} x_{m}^{\beta}|D v|^{2} \mathrm{~d} x .
$$

Now let $t \leq \rho$. Then $t \in\left[\theta_{1}^{k+1} \rho, \theta_{1}^{k} \rho\right]$ for some $k \in \mathbb{N} \cup\{0\}$ and we see that

$$
\begin{aligned}
t^{2-m-\beta} \int_{B_{t}^{+}(y)} x_{m}^{\beta}|D v|^{2} \mathrm{~d} x & \leq\left(\theta_{1}^{k+1} \rho\right)^{2-m-\beta} \int_{B_{\theta_{1}^{k}}(y)} x_{m}^{\beta}|D v|^{2} \mathrm{~d} x \\
& =\theta_{1}^{2-m-\beta}\left(\theta_{1}^{k} \rho\right)^{2-m-\beta} \int_{B_{\theta_{1}^{+} \rho}^{+}(y)} x_{m}^{\beta}|D v|^{2} \mathrm{~d} x \\
& \leq C \frac{\left(\theta_{1}^{k} \rho\right)^{\gamma_{0}}}{\rho^{\gamma_{0}}} \rho^{2-m-\beta} \int_{B_{\rho}^{+}(y)} x_{m}^{\beta}|D v|^{2} \mathrm{~d} x \\
& \leq C\left(\frac{t}{\rho}\right)^{\gamma_{0}} \rho^{2-m-\beta} \int_{B_{\rho}^{+}(y)} x_{m}^{\beta}|D v|^{2} \mathrm{~d} x
\end{aligned}
$$

Now we consider balls in $B_{\rho}(y) \subset B_{R}^{+}\left(x_{0}\right)$ with $\operatorname{dist}\left(B_{\rho}(y) ; \mathbb{R}^{m-1} \times\{0\}\right) \geq \rho$ and $y \in B_{\frac{R}{2}}^{+}\left(x_{0}\right)$. In this case, the Riemannian metric, restricted to $B_{\rho}(y)$ and multiplied by $\rho^{-\frac{\alpha}{2}}$, is uniformly bounded from above and below. We have similar control for 
all the derivatives of this multiple of the metric in $B_{\rho}(y)$. It, hence, follows from the monotonicity inequality, Corollary 4.2, and from [3] and [27] that there is a constant $C$ and a $\gamma_{1}$ which do not depend on $\rho$ or $y$ such that for $t \leq \rho$

$$
t^{2-m} \int_{B_{t}(y)}|D v|^{2} \mathrm{~d} x \leq C\left(\frac{t}{\rho}\right)^{\gamma_{1}} \rho^{2-m} \int_{B_{\rho}(y)}|D v|^{2} \mathrm{~d} x
$$

Setting $\gamma=\min \left\{\gamma_{0}, \gamma_{1}\right\}$ the remainder of the proof follows exactly as in the proof of Theorem 4.21 of [24]; we combine (9.3), (9.4) and an application of a version of Morrey's Decay Lemma adapted to the situation considered here, see [24] Lemma 4.8 , to conclude the proof.

From $\varepsilon$-regularity we deduce partial regularity using a covering argument. Let $\mathscr{H}^{t}$ denote the $t$-dimensional Hausdorff measure. The following is the full version of Theorem 1.1.

Theorem 9.2 Let $\beta \in(-1,1)$. Suppose $v \in W_{\beta, \text { loc }}^{1,2}\left(\mathcal{M} ; \mathbb{S}^{n-1}\right)$ is weakly stationary harmonic with respect to free boundary data. There exists a relatively closed set $\Sigma \subset \mathcal{M}$ with $\mathscr{H}^{m-2}(\Sigma \cap$ int $\mathcal{M})=0$ and $\mathscr{H}^{m-2+\beta}(\Sigma \cap \partial \mathcal{M})=0$ and there exists $\gamma \in(0,1)$ such that $v \in C_{l o c}^{0, \gamma}(\mathcal{M} \backslash \Sigma ; \mathcal{N})$.

Proof It suffices to prove the statement in coordinate patches. Moreover, in the interior of $\mathcal{M}$, the statement follows from the known regularity theory for harmonic maps, in particular [6]. Hence, we may consider $\mathscr{U} \subset \mathbb{R}^{m-1} \times[0, \infty)$ as in Sect. 2 and regard $g$ as a Riemannian metric on $\mathscr{U}$, as we did in the last few sections.

Define

$$
\Sigma_{I}=\left\{x \in \mathscr{U} \cap\left(\mathbb{R}^{m-1} \times(0, \infty)\right): v \text { is not smooth in any neighbourhood of } x\right\} .
$$

By definition $\mathscr{U} \backslash \Sigma_{I}$ is relatively open and the partial regularity theory of Evans [6], implies that $\mathscr{H}^{m-2}\left(\Sigma_{I}\right)=0$.

Now define

$$
\Sigma_{B}=\left\{x \in \mathscr{U} \cap\left(\mathbb{R}^{m-1} \times\{0\}\right): \lim _{\rho \rightarrow 0^{+}} \rho^{2-m-\beta} \int_{B_{\rho}^{+}(y)} x_{m}^{\beta}|D v|^{2} \mathrm{~d} x>0\right\}
$$

and let $\Sigma=\Sigma_{B} \cup \Sigma_{I}$. Let $\partial^{0} \mathscr{U}:=\mathscr{U} \cap\left(\mathbb{R}^{m-1} \times\{0\}\right)$. We show $\mathscr{U} \backslash \Sigma=$ $\mathscr{U} \backslash \Sigma_{I} \cup \partial^{0} \mathscr{U} \backslash \Sigma_{B}$ is relatively open in $\mathscr{U}$. Let $x \in \mathscr{U} \backslash \Sigma$. If $x \in \mathbb{R}^{m-1} \times(0, \infty)$ then by definition $v$ is smooth in a neighbourhood of $x$ and this neighbourhood is therefore contained in $\mathscr{U} \backslash \Sigma_{I} \subset \mathscr{U} \backslash \Sigma$. If $x \in \partial^{0} \mathscr{U} \backslash \Sigma_{B}$ then there exists $R>0$ such that $R^{2-m-\beta} \int_{B_{\rho}^{+}(x)} y_{m}^{\beta}|D v|_{g}^{2} \mathrm{dvol}_{g} \leq \varepsilon_{2}$, where $\varepsilon_{2}$ is the number from Theorem 9.1. Hence, there exists $\theta_{2}, \gamma \in(0,1)$ such that $\left.v \in C^{0, \gamma} \overline{\left(B_{\theta_{2} R}^{+}\left(x_{0}\right)\right.} ; \mathbb{S}^{n-1}\right)$. It then follows from regularity theory for harmonic maps, see e.g [27] Lemma 3.1, that since $v$ is continuous in $B_{\theta_{2} R}^{+}\left(x_{0}\right)$ it is smooth there and, hence, $v$ is smooth in a neighbourhood of any point in $B_{\theta_{2} R}^{+}\left(x_{0}\right)$. Furthermore, it follows from the proof of the theorem, see 
(9.3), that

$$
t^{2-m-\beta} \int_{B_{t}^{+}(y)} x_{m}^{\beta}|D v|^{2} \mathrm{~d} x \leq C\left(\frac{t}{\rho}\right)^{\gamma_{0}} \rho^{2-m-\beta} \int_{B_{\rho}^{+}(y)} x_{m}^{\beta}|D v|^{2} \mathrm{~d} x
$$

for every $B_{\rho}^{+}(y)$ with $\rho \in\left(0, \frac{R}{2}\right]$ and $y \in B_{\frac{R}{2}}\left(x_{0}\right) \cap\left(\mathbb{R}^{m-1} \times\{0\}\right)$ and every $t \leq \rho$. Fixing $\rho=\frac{R}{2}$ we see that

$$
\begin{aligned}
t^{2-m-\beta} \int_{B_{t}^{+}(y)} x_{m}^{\beta}|D v|^{2} \mathrm{~d} x & \leq C\left(\frac{2 t}{R}\right)^{\gamma_{0}}\left(\frac{R}{2}\right)^{2-m-\beta} \int_{B_{\frac{R}{2}}^{+}(y)} x_{m}^{\beta}|D v|^{2} \mathrm{~d} x \\
& \leq C\left(\frac{t}{R}\right)^{\gamma_{0}} \varepsilon_{2}
\end{aligned}
$$

and, hence, every $y \in B_{\frac{R}{2}}\left(x_{0}\right) \cap\left(\mathbb{R}^{m-1} \times\{0\}\right)$ belongs to $\partial^{0} \mathscr{U}$. Setting $\sigma=\min \left\{\frac{1}{2}, \theta_{2}\right\}$ we then see that $\overline{B_{\sigma R}^{+}\left(x_{0}\right)} \subset \mathscr{U} \backslash \Sigma$. Hence, $\Sigma$ is relatively closed. We see that $\mathscr{H}^{m-2+\beta}\left(\Sigma_{B}\right)=0$ using a covering argument analogous to that of the proof of [24] Theorem 4.3.

Acknowledgements James Roberts is grateful for being funded as a Postdoctoral researcher on the Royal Society Grant of Professor Manuel del Pino, with Grant Number: RP R1 180114.

Open Access This article is licensed under a Creative Commons Attribution 4.0 International License, which permits use, sharing, adaptation, distribution and reproduction in any medium or format, as long as you give appropriate credit to the original author(s) and the source, provide a link to the Creative Commons licence, and indicate if changes were made. The images or other third party material in this article are included in the article's Creative Commons licence, unless indicated otherwise in a credit line to the material. If material is not included in the article's Creative Commons licence and your intended use is not permitted by statutory regulation or exceeds the permitted use, you will need to obtain permission directly from the copyright holder. To view a copy of this licence, visit http://creativecommons.org/licenses/by/4.0/.

\section{References}

1. Baird, P., Eells, J.: A conservation law for harmonic maps. In: Geometry Symposium, Utrecht 1980 (Utrecht, 1980), volume 894 of Lecture Notes in Math., pp. 1-25. Springer, Berlin (1981)

2. Baldes, A.: Harmonic mappings with partially free boundary. Manuscr. Math. 40(2-3), 255-275 (1982)

3. Bethuel, F.: On the singular set of stationary harmonic maps. Manuscr. Math. 78(4), 417-443 (1993)

4. Chanillo, S.: Sobolev inequations involving divergence free maps. Commun. Partial Differ. Equ. 16(12), 1969-1994 (1991)

5. Coifman, R.: Pierre-Louis Lions, Yves Meyer, and Stephen Semmes. Compacité par compensation et espaces de hardy. Séminaire Équations aux dérivées partielles (Polytechnique), pp. 1-8, (1990)

6. Evans, L.C.: Partial regularity for stationary harmonic maps into spheres. Arch. Rational Mech. Anal. 116(2), 101-113 (1991)

7. Fabes, E.B., Kenig, C.E., Serapioni, R.P.: The local regularity of solutions of degenerate elliptic equations. Commun. Partial Differ. Equ. 7(1), 77-116 (1982)

8. Giaquinta, M., Martinazzi, L.: An Introduction to the Regularity Theory for Elliptic Systems, Harmonic Maps and Minimal Graphs. Springer, New York (2013)

9. Große-Brauckmann, K.: Interior and boundary monotonicity formulas for stationary harmonic maps. Manuscr. Math. 77(1), 89-95 (1992) 
10. Gulliver, R., Jost, J.: Harmonic maps which solve a free-boundary problem. J. Reine Angew. Math. 381, 61-89 (1987)

11. Heinonen, J., Kilpeläinen, T., Martio, O.: Nonlinear Potential Theory of Degenerate Elliptic Equations. Oxford Mathematical Monographs. The Clarendon Press, Oxford University Press, New York (1993). Oxford Science Publications

12. Hélein, F.: Sur la régularité des applications faiblement harmoniques entre une surface et une variété riemannienne. In Séminaire sur les Équations aux Dérivées Partielles, 1990-1991, pages Exp. No. X, 6. École Polytech., Palaiseau, (1991)

13. Hélein, F.: Regularité des applications faiblement harmoniques entre une surface et une sphere. CR Acad. Sci. Paris Sér. I 311(9), 519-524 (1990)

14. Jost, J.: Riemannian Geometry and Geometric Analysis. Springer, New York (2008)

15. Mazowiecka, K., Schikorra, A.: Fractional div-curl quantities and applications to nonlocal geometric equations. J. Func. Anal. 275(1), 1-44 (2018)

16. Millot, V., Pegon, M., Schikorra, A.: Partial regularity for fractional harmonic maps into spheres. arXiv preprint arXiv:1909.11466 (2019)

17. Millot, V., Sire, Y., Yu, H.: Minimizing fractional harmonic maps on the real line in the supercritical regime. arXiv preprint arXiv:1710.04754 (2017)

18. Moser, R.: Partial Regularity for Harmonic Maps and Related Problems. World Scientific, Singapore (2005)

19. Moser, R.: Intrinsic semiharmonic maps. J. Geom. Anal. 21(3), 588-598 (2011)

20. Nash, J.: The imbedding problem for Riemannian manifolds. Ann. Math. 2(63), 20-63 (1956)

21. Rivière, T.: Everywhere discontinuous harmonic maps into spheres. Acta Math. 175(2), 197-226(1995)

22. Rivière, T.: Conservation laws for conformally invariant variational problems. Invent. Math. 168(1), 1-22 (2007)

23. Riviere, T., Struwe, M.: Partial regularity for harmonic maps and related problems. Commun. Pure Appl. Math. 61(4), 451-463 (2008)

24. Roberts, J.: A regularity theory for intrinsic minimising fractional harmonic maps. Calc. Var. Partial Differ. Equ. 57(4), 109 (2018)

25. Scheven, C.: Partial regularity for stationary harmonic maps at a free boundary. Math. Z. 253(1), 135-157 (2006)

26. Schoen, R., Uhlenbeck, K.: A regularity theory for harmonic maps. J. Differ. Geom. 17(2), 307-335 (1982)

27. Schoen, R.M.: Analytic aspects of the harmonic map problem. In: Seminar on nonlinear partial differential equations (Berkeley, Calif., 1983), volume 2 of Math. Sci. Res. Inst. Publ., pp. 321-358. Springer, New York, (1984)

28. Semmes, S.: A primer on hardy spaces, and some remarks on a theorem of evans and müller. Commun. Partial Differ. Equ. 19(1-2), 277-319 (1994)

29. Simon, L.: Theorems on Regularity and Singularity of Energy Minimizing Maps. Springer, Cham (1996)

30. Sire, Y., Terracini, S., Vita, S.: Liouville type theorems and regularity of solutions to degenerate or singular problems part i: even solutions (2020)

31. Stein, E.M.: Harmonic analysis: real-variable methods, orthogonality, and oscillatory integrals, volume 43 of Princeton Mathematical Series. Princeton University Press, Princeton, NJ, (1993). With the assistance of Timothy S. Murphy, Monographs in Harmonic Analysis, III

32. Strömberg, J.-O., Torchinsky, A.: Weighted Hardy Spaces. Lecture Notes in Mathematics, vol. 1381. Springer, Berlin (1989)

33. Turesson, B.O.: Nonlinear Potential Theory and Weighted Sobolev Spaces. Lecture Notes in Mathematics, vol. 1736. Springer, Berlin (2000)

Publisher's Note Springer Nature remains neutral with regard to jurisdictional claims in published maps and institutional affiliations. 